\title{
ON THE MATHEMATICAL SYNTHESIS OF EQUATIONAL LOGICS*
}

\author{
MARCELO FIORE $^{a}$ AND CHUNG-KIL HUR ${ }^{b}$ \\ ${ }^{a}$ University of Cambridge, Computer Laboratory, \\ 15 JJ Thomson Avenue, Cambridge CB3 0FD, UK. \\ e-mail address: Marcelo.Fiore@cl.cam.ac.uk \\ ${ }^{b}$ Max Planck Institute for Software Systems (MPI-SWS), \\ Kaiserslautern and Saarbrücken, Germany. \\ e-mail address: gil@mpi-sws.org
}

\begin{abstract}
We provide a mathematical theory and methodology for synthesising equational logics from algebraic metatheories. We illustrate our methodology by means of two applications: a rational reconstruction of Birkhoff's Equational Logic and a new equational logic for reasoning about algebraic structure with name-binding operators.
\end{abstract}

\section{$\underline{\text { INTRODUCTION }}$}

Birkhoff (1935) initiated the general study of algebraic structure. Importantly for our concerns here, his development was from (universal) algebra to (equational) logic. Birkhoff's starting point was the informal conception of algebra based on familiar concrete examples. Abstracting from these, he introduced the concepts of signature and equational presentation, and thereby formalised what is now our notion of (abstract) algebra. Subsequently he set up the model theory of equational presentations (varieties) and analysed their structure from the standpoint of logical inference for algebraic languages. In doing so, he introduced Equational Logic as a sound and complete deductive system for reasoning about equational assertions in Algebraic Theories.

Since Birkhoff's work, our understanding of algebraic structure has deepened; having been both systematised and extended (see e.g. Lawvere (1963), Ehresmann (1968), Burroni (1981), Kelly and Power (1993), Power (1999)). On the other hand, the development of equational logics has remained ad hoc. The main aim of the current work is to fill in this gap.

1998 ACM Subject Classification: D.3.1, F.3.1, F.3.2, F.4.1, I.2.3.

Key words and phrases: Equational logic, algebraic theories, soundness and completeness, rewriting, variable binding, $\alpha$-equivalence.

* This paper gives a complete development (with proofs) of results announced in Fiore and Hur (2008). However, for simplicity of exposition, these results are restricted here to the case of mono-sorted algebraic theories and equational logics.

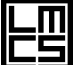

든 IN COMPUTER SCIENCE
DOI:10.2168/LMCS-6 (3:12) 2010

(C) M. Fiore and C.-K. Hur

(c) Creative Commons 
Our standpoint is that equational logics should arise from algebraic structure. In this direction, our first purpose is to provide a mathematical theory and methodology for synthesising equational logics from algebraic metatheories (Part I). Our second purpose is to establish the practicality of the approach. In this respect, we illustrate our methodology by means of two applications: a rational reconstruction of Birkhoff's Equational Logic and a new equational logic for reasoning about algebraic structure with name-binding operators (Part II).

\section{PART I. THEORY}

In this first part of the paper, we present our mathematical framework for synthesising equational logics. For simplicity of exposition, we restrict attention to the mono-sorted context. As such, we consider algebraic metatheories given by strong monads on symmetric monoidal categories. These provide algebraic structure that allows the specification of equational presentations in the form of Monadic Equational Systems (Section 1). Monadic Equational Systems come equipped with a canonical model theory whereby models are Eilenberg-Moore algebras satisfying the equations. An Equational Metalogic (Section 2) for reasoning about equality in such models is presented. This deductive system has been designed to guarantee sound derivations. As for completeness (Section 3), a mathematical justification of the well-known use of free constructions in equational completeness proofs is given, and this is backed up with an inductive method for constructing free algebras.

\section{Monadic Equational Systems}

Monadic Equational Systems (MESs) are defined and their model theory is explained.

1.1. Monadic Equational Systems. The concept of MES provides a general abstract notion of equational presentation.

Definition 1.1 (Terms and equations). A term for an endofunctor $T$ on a category $\mathscr{C}$ of arity $A$ and coarity $C$ is a Kleisli map $C \longrightarrow T A$ in $\mathscr{C}$. A parallel pair of terms $t \equiv t^{\prime}: C \longrightarrow T A$ is called an equation.

Definition 1.2 (Monadic Equational Systems). A $M E S \mathbb{S}=(\underline{\mathscr{C}}, \mathbf{T}, \mathcal{A})$ consists of a strong monad $\mathbf{T}$ on a symmetric monoidal closed category $\underline{\mathscr{C}}$ together with a set of equations $\mathcal{A}$.

Notation. For a strong monad $\mathbf{T}$ on a symmetric monoidal closed category $\mathscr{C}$ we implicitly assume that the respective underlying structures are denoted by $(T, \eta, \mu$, st $)$ and $(\mathscr{C}, I, \otimes,[-,=])$. 
1.2. Model theory. Terms admit interpretations in algebras and these give a modeltheoretic notion of equality.

Let $(T$, st) be a strong endofunctor on a symmetric monoidal closed category $\underline{C}$. Every term $t: C \longrightarrow T A$ admits an interpretation

$$
\llbracket t \rrbracket_{(X, s)}:[A, X] \otimes C \rightarrow X
$$

in a $T$-algebra $(X, s: T X \rightarrow X)$ given by the composite

$$
[A, X] \otimes C \stackrel{[A, X] \otimes t}{\longrightarrow}[A, X] \otimes T A \stackrel{\text { st }_{[A, X], A}}{\longrightarrow} T([A, X] \otimes A) \stackrel{T\left(\epsilon_{X}^{A}\right)}{\longrightarrow} T X \stackrel{s}{\longrightarrow} X .
$$

We thus obtain a satisfaction relation between algebras and equations: for all $T$-algebras $(X, s)$ and equations $u \equiv v: C \longrightarrow T A$,

$$
(X, s) \models u \equiv v: C \rightarrow T A \quad \text { iff } \quad \llbracket u \rrbracket_{(X, s)}=\llbracket v \rrbracket_{(X, s)}:[A, X] \otimes C \rightarrow X .
$$

Definition 1.3 (Algebras). An $\mathbb{S}$-algebra for a $\operatorname{MES} \mathbb{S}=(\underline{\mathscr{C}}, \mathbf{T}, \mathcal{A})$ is an Eilenberg-Moore algebra $(X, s)$ for the monad $\mathbf{T}$ satisfying the equations in $\mathcal{A}$; that is, such that $(X, s) \models u \equiv v: C \longrightarrow T A$ for all $(u \equiv v: C \longrightarrow T A) \in \mathcal{A}$.

The category $\mathbb{S}$-Alg is the full subcategory of the category $\mathscr{C}^{\mathbf{T}}$ (of Eilenberg-Moore algebras for the monad $\mathbf{T}$ ) consisting of the $\mathbb{S}$-algebras. We thus have the following situation

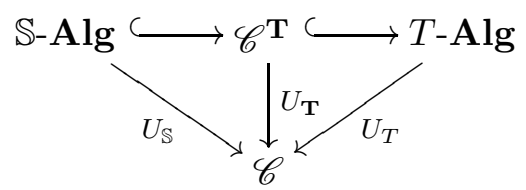

where $T$-Alg denotes the category of algebras for the endofunctor $T$.

\section{Equational Metalogic}

We present a sound deductive system for reasoning about the equality of terms in MESs.

2.1. Equational Metalogic. The Equational Metalogic (EML) associated to a MES $\mathbb{S}=$ $(\underline{\mathscr{C}}, \mathbf{T}, \mathcal{A})$ has judgements of the form

$$
\mathcal{A} \vdash u \equiv v: C \rightarrow T A
$$

where $u$ and $v$ are terms of arity $A$ and coarity $C$, and consists of the following inference rules.

- Equality rules.

$$
\begin{array}{cc}
\operatorname{Ref} \frac{\operatorname{Sym} \frac{\mathcal{A} \vdash u \equiv v: C \longrightarrow T A}{\mathcal{A} \vdash v \equiv u: C \rightarrow T A}}{\mathcal{A} \vdash u \equiv u: C \longrightarrow T A} \quad \\
\operatorname{Trans} \frac{\mathcal{A} \vdash u \equiv v: C \rightarrow T A}{\mathcal{A} \vdash u \equiv w: C \longrightarrow T \equiv w: C \longrightarrow T A}
\end{array}
$$


- Axioms.

$$
\operatorname{Axiom} \frac{(u \equiv v: C \rightarrow T A) \in \mathcal{A}}{\mathcal{A} \vdash u \equiv v: C \longrightarrow T A}
$$

- Congruence of substitution.

$$
\text { Subst } \frac{\mathcal{A} \vdash u_{1} \equiv v_{1}: C \rightarrow T B \quad \mathcal{A} \vdash u_{2} \equiv v_{2}: B \rightarrow T A}{\mathcal{A} \vdash u_{1}\left\{u_{2}\right\} \equiv v_{1}\left\{v_{2}\right\}: C \longrightarrow T A}
$$

where $w_{1}\left\{w_{2}\right\}$ denotes the Kleisli composite $C \stackrel{w_{1}}{\longrightarrow} T B \stackrel{T\left(w_{2}\right)}{\longrightarrow} T(T A) \stackrel{\mu_{A}}{\longrightarrow} T A$.

- Congruence of tensor extension.

$$
\operatorname{Ext} \frac{\mathcal{A} \vdash u \equiv v: C \rightarrow T A}{\mathcal{A} \vdash\langle V\rangle u \equiv\langle V\rangle v: V \otimes C \rightarrow T(V \otimes A)}
$$

where $\langle V\rangle w$ denotes the composite $V \otimes C \stackrel{V \otimes w}{\longrightarrow} V \otimes T A \stackrel{\text { st }_{V, A}}{\longrightarrow} T(V \otimes A)$.

- Local character.

$$
\text { Local } \frac{\mathcal{A} \vdash u \circ e_{i} \equiv v \circ e_{i}: C_{i} \rightarrow T A \quad(i \in I)}{\mathcal{A} \vdash u \equiv v: C \longrightarrow T A}\left(\left\{e_{i}: C_{i} \rightarrow C\right\}_{i \in I} \text { jointly epi }\right)
$$

(Recall that a family of maps $\left\{e_{i}: C_{i} \longrightarrow C\right\}_{i \in I}$ is said to be jointly epi if, for any $f, g: C \longrightarrow X$ such that $\forall_{i \in I} f \circ e_{i}=g \circ e_{i}: C_{i} \longrightarrow X$, it follows that $f=g$.)

Remark 2.1. In the presence of coproducts and under the rule Ref, the rules Subst and Local are inter-derivable with the rules

$$
\text { Subst }_{\amalg} \frac{\mathcal{A} \vdash u \equiv v: C \longrightarrow T\left(\coprod_{i \in I} B_{i}\right) \quad \mathcal{A} \vdash u_{i} \equiv v_{i}: B_{i} \longrightarrow T A(i \in I)}{\mathcal{A} \vdash u\left\{\left[u_{i}\right]_{i \in I}\right\} \equiv v\left\{\left[v_{i}\right]_{i \in I}\right\}: C \longrightarrow T A}
$$

and

$$
\operatorname{Local}_{1} \frac{\mathcal{A} \vdash u \circ e \equiv v \circ e: C^{\prime} \longrightarrow T A}{\mathcal{A} \vdash u \equiv v: C \longrightarrow T A}\left(e: C^{\prime} \rightarrow C \text { epi }\right)
$$

Indeed, consider the rule

$$
\operatorname{Local}_{\amalg} \frac{\mathcal{A} \vdash u_{i} \equiv v_{i}: C_{i} \longrightarrow T A \quad(i \in I)}{\mathcal{A} \vdash\left[u_{i}\right]_{i \in I} \equiv\left[v_{i}\right]_{i \in I}: \coprod_{i \in I} C_{i} \longrightarrow T A}
$$

and note that: $(i)$ the rule Local is derivable from the rules Local ${ }_{1}$ and Local ${ }_{\amalg}$, which are in turn instances of the rule Local; $(i i)$ the rule Subst $\amalg$ is derivable from the rules Subst and Local $_{\amalg}$; $(i i i)$ the rule Subst is an instance of the rule Subst $\amalg$; and $(i v)$ assuming the rule Ref, the rule Local $_{\amalg}$ is derivable from the rule Subst ${ }_{\amalg}$. 
2.2. Soundness. The following result states the soundness of derivability in EML. We write $\mathbb{S}$-Alg $\models u \equiv v: C \rightarrow T A$ whenever $(X, s) \models u \equiv v: C \rightarrow T A$ for all $\mathbb{S}$-algebras $(X, s)$.

Theorem 2.2 (Soundness). For a $\operatorname{MES} \mathbb{S}=(\underline{\mathscr{C}}, \mathbf{T}, \mathcal{A})$,

$$
\mathcal{A} \vdash u \equiv v: C \longrightarrow T A \quad \text { implies } \quad \mathbb{S}-\mathbf{A l g} \models u \equiv v: C \rightarrow T A .
$$

Proof. See Appendix A,

\section{Internal Completeness}

In this section we build a mathematical basis for investigating completeness. Our main tools are an internal completeness result for MESs that admit free algebras together with an inductive method for constructing them.

3.1. Internal Completeness. Let $\mathbb{S}=(\underline{\mathscr{C}}, \mathbf{T}, \mathcal{A})$ be a MES admitting free algebras; that is, such that the forgetful functor $U_{\mathbb{S}}: \mathbb{S}-\mathbf{A l g} \rightarrow \mathscr{C}$ has a left adjoint. We denote the free $\mathbb{S}$-algebra on $X \in \mathscr{C}$ as $\left(T_{\mathbb{S}} X, \tau_{X}^{\mathbb{S}}: T T_{\mathbb{S}} X \rightarrow T_{\mathbb{S}} X\right)$, and the associated free $\mathbb{S}$-algebra monad as $\mathbf{T}_{\mathbb{S}}=\left(T_{\mathbb{S}}, \eta^{\mathbb{S}}, \mu^{\mathbb{S}}\right)$. Then, the embedding $\mathbb{S}$-Alg $\hookrightarrow \mathscr{C}^{\mathbf{T}}$ induces a strong monad morphism $\mathrm{q}^{\mathbb{S}}: \mathbf{T} \longrightarrow \mathbf{T}_{\mathbb{S}}$. This has components referred to as quotient maps that are characterised by being the unique morphisms $\mathrm{q}_{X}^{\mathbb{S}}: T X \rightarrow T_{\mathbb{S}} X$ for which the diagram

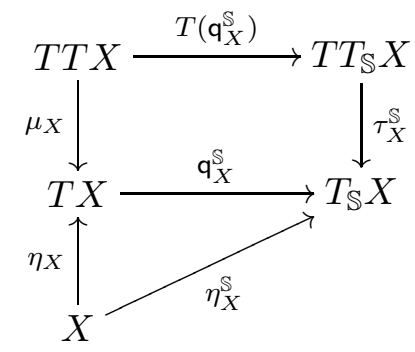

commutes. In this situation, we have a form of strong completeness stating that an equation is satisfied in all models if and only if it is satisfied in a freely generated one, if and only if it is identified by the quotient map.

Theorem 3.1 (Internal completeness). For a $M E S \mathbb{S}=(\underline{\mathscr{C}}, \mathbf{T}, \mathcal{A})$ admitting free algebras, the following are equivalent.

(1) $\mathbb{S}$-Alg $\models u \equiv v: C \rightarrow T A$.

$(2)\left(T_{\mathbb{S}} A, \tau_{A}^{\mathbb{S}}\right) \models u \equiv v: C \rightarrow T A$.

$(3) \mathrm{q}_{A}^{\mathbb{S}} \circ u=\mathrm{q}_{A}^{\mathbb{S}} \circ v: C \rightarrow T_{\mathbb{S}} A$.

Proof. See Appendix B, 
3.2. Free Algebras. We now establish a general setting in which to apply the internal completeness theorem. Indeed, we give conditions under which MESs admit free algebras and provide an inductive construction of quotient maps (see Fiore and Hur (2009) for details).

Definition 3.2. Let $\mathscr{C}$ be a symmetric monoidal closed category. An object $A$ in $\mathscr{C}$ is respectively said to be compact and projective if the endofunctor $[A,-]$ on $\mathscr{C}$ respectively preserves colimits of $\omega$-chains and epimorphisms.

Definition 3.3. A MES $\mathbb{S}=(\mathscr{C}, \mathbf{T}, \mathcal{A})$ is called finitary if the category $\mathscr{C}$ is cocomplete, the endofunctor $T$ on $\mathscr{C}$ is $\omega$-cocontinuous, and the arity $A$ of each equation $u \equiv v: C \rightarrow T A$ in $\mathcal{A}$ is compact. Such a MES is called inductive if furthermore the endofunctor $T$ preserves epimorphisms and the arity $A$ of each equation $u \equiv v: C \rightarrow T A$ in $\mathcal{A}$ is projective.

For a finitary $\operatorname{MES} \mathbb{S}=(\underline{\mathscr{C}}, \mathbf{T}, \mathcal{A})$ we have the following situation:

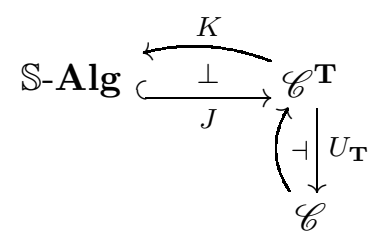

For each object $X \in \mathscr{C}$, since $\left(T X, \mu_{X}\right)$ is a free Eilenberg-Moore algebra on $X$, the free $\mathbb{S}$-algebra $\left(T_{\mathbb{S}} X, \tau_{X}^{\mathbb{S}}\right)$ on $X$ is given by the free $\mathbb{S}$-algebra $K\left(T X, \mu_{X}\right)$ over the EilenbergMoore algebra $\left(T X, \mu_{X}\right)$. Satisfying the commutative diagram (3.1), the universal homomorphism $\left(T X, \mu_{X}\right) \rightarrow\left(T_{\mathbb{S}} X, \tau_{X}^{\mathbb{S}}\right)$ induced by the adjunction $K \dashv J$ yields the quotient $\operatorname{map} \mathrm{q}_{X}^{\mathbb{S}}: T X \rightarrow T_{\mathbb{S}} X$.

In the case of inductive MESs, the quotient maps $q_{X}^{\mathbb{S}}$ are constructed as follows:

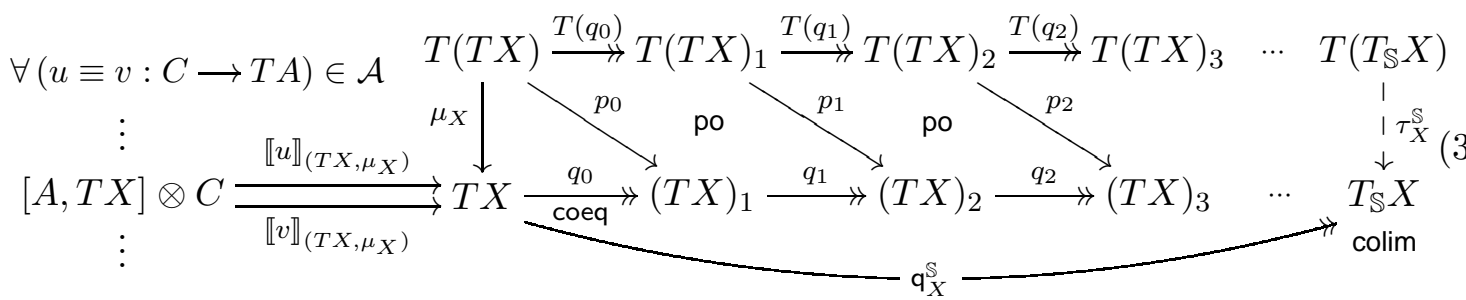

where $q_{0}$ is the universal map that jointly coequalizes every pair $\llbracket u \rrbracket_{\left(T X, \mu_{X}\right)}$ and $\llbracket v \rrbracket_{\left(T X, \mu_{X}\right)}$ with $(u \equiv v: C \rightarrow T A) \in \mathcal{A}$ and where, for all $n \geq 1$, the cospans

$$
(T X)_{n} \stackrel{q_{n}}{\longrightarrow}(T X)_{n+1} \stackrel{p_{n}}{\longleftarrow} T(T X)_{n}
$$

are pushouts of the spans

$$
(T X)_{n} \stackrel{p_{n-1}}{\longleftarrow} T(T X)_{n-1} \stackrel{T\left(q_{n-1}\right)}{\longleftrightarrow} T(T X)_{n}
$$

for $(T X)_{0}=T X$. 
Moreover, when the strong monad $\mathbf{T}$ arises from a left adjoint to a forgetful functor $F$-Alg $\longrightarrow \mathscr{C}$, for $F$ a strong endofunctor that preserves colimits of $\omega$-chains and epimorphisms, the construction of the quotient maps $\mathrm{q}_{X}^{\mathbb{S}}$ simplifies as follows:

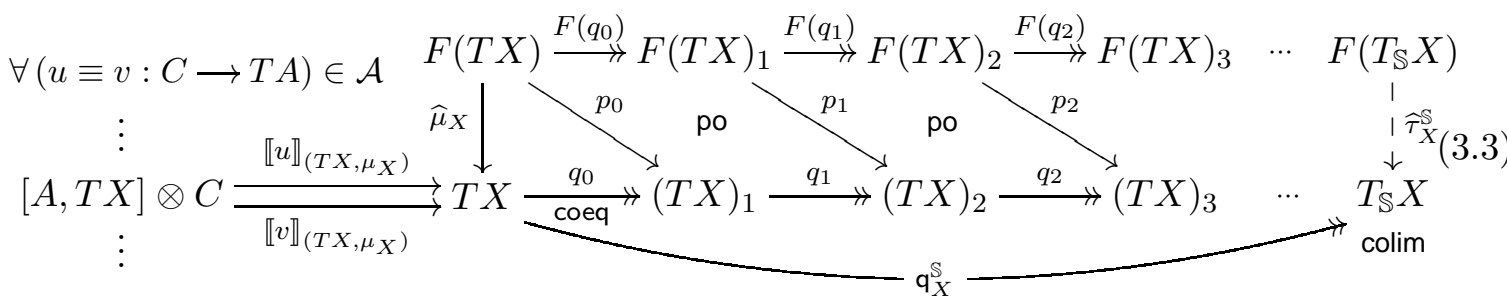

where $\left(T X, \widehat{\mu}_{X}\right)$ and $\left(T_{\mathbb{S}} X, \widehat{\tau}_{X}^{\mathbb{S}}\right)$ are the $F$-algebras respectively corresponding to the EilenbergMoore algebras $\left(T X, \mu_{X}\right)$ and $\left(T_{\mathbb{S}} X, \tau_{X}^{\mathbb{S}}\right)$ for the monad $\mathbf{T}$. (Explicit calculations of this construction feature in Sections 4.6 and 5.7.)

\section{PART II. MeThodology}

In view of the mathematical development of Part I, we advocate the following methodology for synthesising mono-sorted equational logics.

(1) Select a symmetric monoidal closed category $\underline{\mathscr{C}}$ as universe of discourse and consider within it a syntactic notion of signature such that every signature $\Sigma$ gives rise to a strong monad $\mathbf{T}_{\Sigma}$ on $\underline{\mathscr{C}}$.

The universe of discourse should be carefully chosen to consist of mathematical objects with enough internal structure to allow for the algebraic realisation of the syntactic constructs that one is modelling.

We do not insist on an a priori prescription for the definition of signature, but rather consider it as being domain specific. Of course, standard notions of signature $(e . g$. as in enriched algebraic theories - see Kelly and Power (1993), and Robinson (2002)) may be considered. However, one may need to go beyond them - see Fiore (2008) and Fiore and Hur (2010).

(2) Select a class of coarity-arity pairs $(C, A)$ of objects of $\mathscr{C}$ and give a syntactic description of Kleisli maps $C \longrightarrow T_{\Sigma} A$. This yields a syntactic notion of equational presentation with an associated model theory arising from that of MESs.

We are ultimately interested in constructing free algebras for equational presentations. In the context of finitary algebraic theories, it is thus appropriate to consider a cocomplete universe of discourse together with signatures for which the associated monad preserves colimits of $\omega$-chains and epimorphisms, and arities that are compact and projective; so that the induced MESs are inductive.

(3) Synthesise a deductive system for equational reasoning on syntactic terms with rules arising as syntactic counterparts of the EML rules associated to the MES.

The analysis of the rule Subst will typically involve the consideration of a syntactic substitution operation corresponding to Kleisli composition. 
(4) Analyse the inductive construction of free algebras and obtain an intermediate deductive system characterising the equivalence induced by the quotient maps. Embed the intermediate deductive system within the synthesised equational logic and conclude the completeness of the latter as a consequence of the internal completeness result.

In practise, we have found that the intermediate deductive system is not only easily embeddable in the synthesised equational logic but that it moreover allows one to distil a rewriting-style deduction system that provides a sound and complete computational treatment of derivability.

The resulting equational logics are thus synthesised from algebraic metatheories by means of first principles. Two sample applications of this methodology follow.

\section{Synthetic Equational Logic}

4.1. MESs for algebraic theories. Recall that an algebraic theory $\mathbb{T}=(\Sigma, E)$ is given by a signature $\Sigma$, consisting of a set of operators $O$ and an arity function $|-|: O_{\Sigma} \rightarrow \mathbb{N}$, together with a set of equations $E$. Algebraic theories may be encoded as MESs as follows.

The signature $\Sigma$ induces the endofunctor $F_{\Sigma}(X)=\coprod_{\mathrm{o} \in O} X^{|\mathrm{o}|}$ on Set, for which the category of $\Sigma$-algebras, $\Sigma$-Alg, and the category of $F_{\Sigma}$-algebras, $F_{\Sigma}$-Alg, are isomorphic. The forgetful functor $F_{\Sigma}$-Alg $\rightarrow$ Set is monadic and the induced term monad $\mathbf{T}_{\Sigma}=$ $\left(T_{\Sigma}, \eta^{\Sigma}, \mu^{\Sigma}\right)$ is given syntactically. For a set of variables $V$, the set $T_{\Sigma}(V)$ consists of terms built up from the variables in $V$ and the operators in $O$.

The endofunctor $F_{\Sigma}$ has a canonical strength st : $U \times F_{\Sigma}(V) \longrightarrow F_{\Sigma}(U \times V)$ mapping a pair $\left(u, \iota_{\mathrm{o}}\left(v_{1}, \ldots, v_{|\mathrm{o}|}\right)\right)$ to $\iota_{\mathrm{o}}\left(\left(u, v_{1}\right), \ldots,\left(u, v_{|\mathrm{o}|}\right)\right)$, where we use the notation $\iota$ for coproduct injections. The induced strength on the monad $\mathbf{T}_{\Sigma}$, $\widehat{\text { st }}: U \times T_{\Sigma}(V) \rightarrow T_{\Sigma}(U \times V)$, maps a pair $(u, t)$ to the term $t\{v \longmapsto(u, v)\}_{v \in V}$ obtained by simultaneously substituting $(u, v)$ for each variable $v \in V$ in the term $t$.

By definition, each equation $(V \vdash l \equiv r)$ in $E$ is given by a pair of terms $l, r \in T_{\Sigma}(V)$, or equivalently, by a parallel pair of Kleisli maps $\underline{l}, \underline{r}: 1 \rightarrow T_{\Sigma}(V)$. Thus, one can encode the algebraic theory $\mathbb{T}$ as the MES $\mathbb{T}=\left(\right.$ Set, $\left.\mathbf{T}_{\Sigma}, \underline{E}\right)$ with the set of equations $\underline{E}$ given by $\left\{\underline{l} \equiv \underline{r}: 1 \longrightarrow T_{\Sigma} V \mid(V \vdash l \equiv r) \in E\right\}$. The MES $\underline{\mathbb{T}}$ is inductive.

4.2. Model theory. A $\underline{\mathbb{T}}$-algebra is an Eilenberg-Moore algebra $\left(X, s: T_{\Sigma} X \rightarrow X\right)$ for $\mathbf{T}_{\Sigma}$ such that the diagram

$$
X^{V} \times 1 \stackrel{X^{V} \times \underline{t_{1}}}{X^{V} \times \underline{t_{2}}} X^{V} \times T_{\Sigma} V \stackrel{\widehat{\mathrm{st}}_{X}{ }^{V}, V}{\longrightarrow} T_{\Sigma}\left(X^{V} \times V\right) \stackrel{T_{\Sigma}\left(\epsilon_{X}^{V}\right)}{\longrightarrow} T_{\Sigma} X \stackrel{s}{\longrightarrow} X
$$

commutes for every equation $\left(V \vdash t_{1} \equiv t_{2}\right)$ in $E$; that is, such that

$$
1 \stackrel{\underline{t_{1}}}{\underset{t_{2}}{\longrightarrow}} T_{\Sigma} V \stackrel{T_{\Sigma}(v)}{\longrightarrow} T_{\Sigma} X \stackrel{s}{\longrightarrow} X
$$

commutes for all functions $v: V \rightarrow X$.

Write $(X, \llbracket-\rrbracket)$ for the Eilenberg-Moore algebra of the monad $\mathbf{T}_{\Sigma}$ corresponding to the $\Sigma$-algebra $\left(X,\{\llbracket \mathrm{o} \rrbracket\}_{\mathrm{o} \in \Sigma}\right)$ via the isomorphism $\Sigma$-Alg $\cong \mathscr{C}^{\mathbf{T}_{\Sigma}}$. We have that the EilenbergMoore algebra $(X, \llbracket-\rrbracket)$ satisfies (4.1) if and only if the $\Sigma$-algebra $\left(X,\{\llbracket \circ \rrbracket\}_{\mathrm{o} \in \Sigma}\right)$ satisfies the 
equation $\left(V \vdash t_{1} \equiv t_{2}\right)$. It follows thus that $\mathbb{T}$-Alg is isomorphic to the category $\mathbb{T}$-Alg of algebras for the algebraic theory $\mathbb{T}$.

4.3. EML for algebraic theories. The EML associated to the MES of an algebraic theory $\mathbb{T}=(\Sigma, E)$ has judgements of the form

$$
\underline{E} \vdash f \equiv g: U \longrightarrow T_{\Sigma} V
$$

with inference rules Ref, Sym, Trans, Axiom, Subst ${ }_{\amalg}$, Ext, and Local ${ }_{1}$ (see Section 2). The rules Ext and Local 1 are however redundant. Indeed, the subsystem $\mathrm{EML}_{1}$ with inference rules Ref, Sym, Trans, Axiom, and Subst $\amalg$ restricted to judgements of the form

$$
\underline{E} \vdash u \equiv v: 1 \rightarrow T_{\Sigma} V
$$

is such that

$$
\text { iff } \begin{aligned}
& \underline{E} \vdash f \equiv g: U \rightarrow T_{\Sigma} V \text { is derivable in EML } \\
& \underline{E} \vdash f\{\underline{i}\} \equiv g\{\underline{i}\}: 1 \longrightarrow T_{\Sigma} V \text { is derivable in } \mathrm{EML}_{1} \text { for all } i \in U .
\end{aligned}
$$

4.4. Synthetic Equational Logic. A Synthetic Equational Logic (SEL) for algebraic theories $\mathbb{T}=(\Sigma, E)$ directly arises as the syntactic counterpart of $\mathrm{EML}_{1}$. SEL has judgements

$$
V \vdash_{E} s \equiv t \quad\left(s, t \in T_{\Sigma} V\right)
$$

and consists of the following rules:

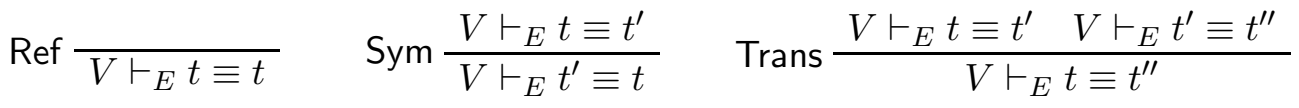

$$
\begin{aligned}
& \text { Axiom } \frac{(V \vdash l \equiv r) \in E}{V \vdash_{E} l \equiv r} \\
& \text { Subst } \frac{U \vdash_{E} t \equiv t^{\prime} \quad V \vdash_{E} s_{u} \equiv s_{u}^{\prime}(u \in U)}{V \vdash_{E} t\left\{u \longmapsto s_{u}\right\}_{u \in U} \equiv t^{\prime}\left\{u \longmapsto s_{u}^{\prime}\right\}_{u \in U}}
\end{aligned}
$$

In the rule Subst, the term $t\left\{u \longmapsto s_{u}\right\}_{u \in U}$ is obtained by simultaneously substituting the terms $s_{u}$ for the variables $u \in U$ in the term $t$.

4.5. Soundness. Note that SEL subsumes the usual presentation of Equational Logic, where the substitution rule is restricted to families $s_{u}=s_{u}^{\prime}(u \in U)$ and a congruence rule for operators is added. Furthermore, since $V \vdash_{E} s \equiv t$ is derivable in SEL iff $\underline{E} \vdash \underline{s} \equiv \underline{t}$ : $1 \longrightarrow T_{\Sigma} V$ is derivable in $\mathrm{EML}_{1}$ iff $\underline{E} \vdash \underline{s} \equiv \underline{t}: 1 \longrightarrow T_{\Sigma} V$ is derivable in EML, the well-known soundness of SEL follows from the soundness of EML. 
4.6. Completeness. We proceed to show how the internal completeness theorem and the construction of free algebras for inductive MESs (see Section 3) lead to equational derivability and bidirectional rewriting completeness results.

Consider the construction (3.3) for the MES $\mathbb{T}$. The map $q_{0}: T_{\Sigma} X \rightarrow\left(T_{\Sigma} X\right)_{1}$ is the universal map in Set that coequalizes every pair $\llbracket l \rrbracket, \llbracket r \rrbracket:\left(T_{\Sigma} X\right)^{V} \rightarrow T_{\Sigma} X$ for all $(V \vdash l \equiv r) \in E$, where $\llbracket t \rrbracket$ maps $s \in\left(T_{\Sigma} X\right)^{V}$ to $t\left\{v \mapsto s_{v}\right\}_{v \in V}$. It follows that the set $\left(T_{\Sigma} X\right)_{1}$ is given by the quotient $T_{\Sigma} X / \approx_{1}$ of $T_{\Sigma} X$ under the equivalence relation $\approx_{1}$ generated by the rule:

$$
\frac{(V \vdash l \equiv r) \in E}{l\left\{v \longmapsto s_{v}\right\}_{v \in V} \approx_{1} r\left\{v \longmapsto s_{v}\right\}_{v \in V}}\left(s \in\left(T_{\Sigma} X\right)^{V}\right)
$$

The map $q_{0}$ sends a term $t \in T_{\Sigma} X$ to its equivalence class $[t]_{\approx_{1}} \in T_{\Sigma} X / \approx_{1}$, and the map $p_{0}$ sends $\iota_{\mathrm{o}}\left(t_{1}, \ldots, t_{|\mathrm{o}|}\right) \in F_{\Sigma}\left(T_{\Sigma} X\right)$ to $\left[\mathrm{o}\left(t_{1}, \ldots, t_{|\mathrm{o}|}\right)\right]_{\approx_{1}} \in T_{\Sigma} X / \approx_{1}$.

Recall that a pushout

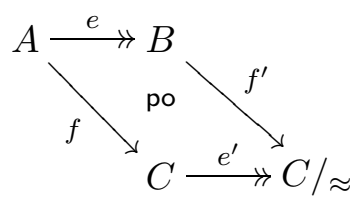

of a surjective map $e: A \rightarrow B$ and a map $f: A \rightarrow C$ in Set can be constructed as the cospan $e^{\prime}: C \rightarrow C / \approx \leftarrow B: f^{\prime}$, where $C / \approx$ is the quotient of $C$ under the equivalence relation $\approx$ generated by setting $f(a) \approx f\left(a^{\prime}\right)$ for all $a, a^{\prime} \in A$ such that $e(a)=e\left(a^{\prime}\right)$ in $B$, and where the surjective map $e^{\prime}: C \rightarrow C / \approx$ sends an element $c$ to its equivalence class $[c]_{\approx}$ and the map $f^{\prime}: B \rightarrow C / \approx$ sends an element $b$ to $e^{\prime}(f(a))$ for $a \in A$ such that $e(a)=b$.

Using this construction, an inductive analysis of the maps $q_{n}$ for $n \geq 1$ shows that the sets $\left(T_{\Sigma} X\right)_{n}$ for $n \geq 2$ are given as the quotients $T_{\Sigma} X / \approx_{n}$ of $T_{\Sigma} X$ under the equivalence relations $\approx_{n}$ inductively generated by the following rules:

$$
\frac{s \approx_{n-1} s^{\prime}}{s \approx_{n} s^{\prime}} \quad \frac{s_{1} \approx_{n-1} s_{1}^{\prime}, \ldots, s_{|\mathrm{o}|} \approx_{n-1} s_{|\mathrm{o}|}^{\prime}}{\mathrm{o}\left(s_{1}, \ldots, s_{|\mathrm{o}|}\right) \approx_{n} \mathrm{O}\left(s_{1}^{\prime}, \ldots, s_{|\mathrm{o}|}^{\prime}\right)}(\mathrm{o} \in \Sigma)
$$

The maps $q_{n}$ for $n \geq 1$ send $[t]_{\approx_{n}} \in T_{\Sigma} X / \approx_{n}$ to $[t]_{\approx_{n+1}} \in T_{\Sigma} X / \approx_{n+1}$, and the maps $p_{n}$ for $n \geq 1$ send $\iota_{\mathrm{o}}\left(\left[t_{1}\right]_{\approx_{n}}, \ldots,\left[t_{|\mathrm{o}|}\right]_{\approx_{n}}\right) \in F_{\Sigma}\left(T_{\Sigma} X / \approx_{n}\right)$ to $\left[\mathrm{o}\left(t_{1}, \ldots, t_{|\mathrm{o}|}\right)\right]_{\approx_{n+1}} \in T_{\Sigma} X / \approx_{n+1}$.

By taking the colimit of the chain of quotients $\left\{q_{n}: T_{\Sigma} X / \approx_{n} \rightarrow T_{\Sigma} X / \approx_{n+1}\right\}_{n \geq 0}$, the set $T_{\mathbb{S}} X$ is given by the quotient $T_{\Sigma} X / \approx_{E}$ of $T_{\Sigma} X$ under the relation $\approx_{E}$ generated by the following rules:

$$
\begin{gathered}
\operatorname{Ref} \frac{\operatorname{Sym} \frac{s \approx_{E} s^{\prime}}{s^{\prime} \approx_{E} s} \quad \operatorname{Trans} \frac{s \approx_{E} s^{\prime} s^{\prime} \approx_{E} s^{\prime \prime}}{s \approx_{E} s^{\prime \prime}}}{\operatorname{Inst} \frac{(V \vdash l \equiv r) \in E}{l\left\{v \longmapsto s_{v}\right\}_{v \in V} \approx_{E} r\left\{v \longmapsto s_{v}\right\}_{v \in V}}\left(s \in\left(T_{\Sigma} X\right)^{V}\right)} \\
\operatorname{Cong} \frac{s_{1} \approx_{E} s_{1}^{\prime}, \ldots, s_{|\mathrm{o}|} \approx_{E} s_{|\mathrm{o}|}^{\prime}}{\mathrm{o}\left(s_{1}, \ldots, s_{|\mathrm{o}|}\right) \approx_{E} \mathrm{O}\left(s_{1}^{\prime}, \ldots, s_{|\mathrm{o}|}^{\prime}\right)}(\mathrm{o} \in \Sigma)
\end{gathered}
$$

The quotient map $\mathrm{q}_{X}^{\mathbb{S}}: T_{\Sigma} X \rightarrow T_{\mathbb{S}} X$ sends a term $t$ to its equivalence class $[t]_{\approx_{E}}$. 
The rules Inst and Cong for the relation $\approx_{E}$ can be merged into a single rule to yield a rewriting-style deduction system. Indeed, by an induction on the depth of proof trees, one shows that the relation $\approx_{E}$ on $T_{\Sigma} X$ coincides with the equivalence relation $\approx_{E}^{\mathrm{R}}$ on $T_{\Sigma} X$ generated by the rewriting-style rule

$$
\mathbf{C}\left[l\left\{v \longmapsto s_{v}\right\}_{v \in V}\right] \quad \approx_{E}^{\mathrm{R}} \mathbf{C}\left[r\left\{v \longmapsto s_{v}\right\}_{v \in V}\right]
$$

for $(V \vdash l \equiv r) \in E, s \in\left(T_{\Sigma} X\right)^{V}$, and $\mathbf{C}[-]$ a context with one hole and possibly with variables from $X$.

From the internal completeness of the MES $\mathbb{T}$, we have the soundness and completeness of equational reasoning by bidirectional rewriting:

$$
\begin{aligned}
\mathbb{T}-A \lg \models(V \vdash s \equiv t) & \\
& \Longleftrightarrow \underline{\mathbb{T}}-\mathbf{A l g}=\left(\underline{s} \equiv \underline{t}: 1 \rightarrow T_{\Sigma} V\right) \\
& \Longleftrightarrow \mathrm{q}_{V}^{\mathbb{S}} \circ \underline{s}=\mathrm{q}_{V}^{\mathbb{S}} \circ \underline{t}: 1 \rightarrow T_{\mathbb{S}} V \\
& \Longleftrightarrow[s]_{\approx_{E}}=[t]_{\approx_{E}} \quad \text { in } T_{\Sigma} V / \approx_{E} \\
& \Longleftrightarrow s \approx_{E} t \quad \text { in } T_{\Sigma} V \\
& \Longleftrightarrow s \approx_{E}^{\mathrm{R}} t \quad \text { in } T_{\Sigma} V
\end{aligned}
$$

Finally, also SEL is complete; as a proof of $s \approx_{E} t$ for $s, t \in T_{\Sigma} V$ constructed by the rules in (4.2) can be turned into a proof of $V \vdash_{E} s \equiv t$ in SEL.

\section{Synthetic Nominal Equational Logic}

This section provides a novel application of our theory and methodology for synthesising equational logics geared to the development of a deductive system for reasoning about algebraic structure with name-binding operators.

We consider a class of MESs, referred to as Nominal Equational Systems (NESs), based on the category Nom of nominal sets (Gabbav and Pitts, 2001, Section 6) (or equivalently the Schanuel topos (Mac Lane and Moerdijk, 1992, Section III.9)). These we subsequently present in syntactic form to yield Nominal Equational Presentations (NEPs). The model theory of NEPs is of course derived from that of NESs.

An equational logic, called Synthetic Nominal Equational Logic (SNEL), for NEPs is derived from the EML associated to NESs. This is guaranteed to be sound by construction. Completeness is derived from the internal completeness theorem by an analysis of the inductive construction of free algebras in terms of equational derivability. This approach yields two completeness results: the rewriting completeness of an induced notion of Synthetic Nominal Rewriting (SNR) and the derivability completeness of SNEL.

A brief discussion of related work is included.

5.1. Nominal sets. For a fixed countably infinite set $A$ of atoms, the group $\mathfrak{S}_{0}(A)$ of finite permutations of atoms consists of the bijections on $A$ that fix all but finitely many elements of $\mathrm{A}$. A $\mathfrak{S}_{0}(\mathrm{~A})$-action $X=(|X|, \cdot)$ consists of a set $|X|$ equipped with a function $(-) \cdot(=): \mathfrak{S}_{0}(\mathrm{~A}) \times|X| \longrightarrow|X|$ satisfying $\mathrm{id}_{\mathrm{A}} \cdot x=x$ and $\pi^{\prime} \cdot(\pi \cdot x)=\left(\pi^{\prime} \pi\right) \cdot x$ for all $x \in|X|$ and $\pi, \pi^{\prime} \in \mathfrak{S}_{0}(\mathrm{~A})$. $\mathfrak{S}_{0}(\mathrm{~A})$-actions form a category with morphisms $X \rightarrow Y$ given by equivariant functions; that is, functions $f:|X| \rightarrow|Y|$ such that $f(\pi \cdot x)=\pi \cdot(f x)$ for all $\pi \in \mathfrak{S}_{0}(\mathrm{~A})$ and $x \in|X|$. 
For a $\mathfrak{S}_{0}(\mathrm{~A})$-action $X$, a finite subset $S$ of $\mathrm{A}$ is said to support $x \in X$ if for all atoms $a, a^{\prime} \notin S$, we have that $\left(a a^{\prime}\right) \cdot x=x$, where the transposition $\left(a a^{\prime}\right)$ is the bijection that swaps $a$ and $a^{\prime}$, and fixes all other atoms. A nominal set is a $\mathfrak{S}_{0}(\mathrm{~A})$-action in which every element has finite support. As an example, the set of atoms A becomes the nominal set of atoms $\mathbb{A}$ when equipped with the evaluation action $\pi \cdot a=\pi(a)$. The category Nom is the full subcategory of the category of $\mathfrak{S}_{0}(\mathrm{~A})$-actions consisting of nominal sets.

The supports of an element of a nominal set are closed under intersection, and we write $\operatorname{supp}_{X}(x)$, or simply supp $(x)$, for the intersection of the supports of $x$ in the nominal set $X$. For instance, we have that $\operatorname{supp}_{\mathbb{A}}(a)=\{a\}$. For elements $x$ and $y$ of two, possibly distinct, nominal sets $X$ and $Y$, we write $x \# y$ whenever $\operatorname{supp}_{X}(x)$ and $\operatorname{supp}_{Y}(y)$ are disjoint. Thus, for $a \in \mathbb{A}$ and $x \in X, a \# x$ stands for $a \notin \operatorname{supp}_{X}(x)$; that is, $a$ is fresh for $x$.

The category Nom is complete and cocomplete. In particular, for a possibly infinite family of nominal sets $\left\{X_{i}\right\}_{i \in I}$, the coproduct $\coprod_{i \in I} X_{i}$ is given by $\left|\coprod_{i \in I} X_{i}\right|=\coprod_{i \in I}\left|X_{i}\right|$ with action $\pi \cdot \iota_{i}(x)=\iota_{i}(\pi \cdot x)$, where we use the notation $\iota$ for coproduct injections; the product $\prod_{i \in I} X_{i}$, for a finite set $I$, is given by $\left|\prod_{i \in I} X_{i}\right|=\prod_{i \in I}\left|X_{i}\right|$ with action $\pi \cdot\left\{x_{i}\right\}_{i \in I}=$ $\left\{\pi \cdot x_{i}\right\}_{i \in I}$. As usual, we write $X^{n}$ for the $n$-fold product $X \times \cdots \times X$.

Further, Nom carries a symmetric monoidal closed structure $(1, \#,[-,=])$. The unit 1 is the terminal object in Nom (i.e., the singleton set consisting of the empty tuple equipped with the unique action). The separating tensor $X \# Y$ is the nominal subset of $X \times Y$ with underlying set given by $\{(x, y) \in|X| \times|Y| \mid x \# y\}$. We write $X^{\# n}$ for the $n$-fold tensor product $X \# \cdots \# X$. For instance, $\mathbb{A}^{\# n}$ consists of $n$-tuples of distinct atoms equipped with the pointwise action $\pi \cdot\left(a_{1}, \ldots, a_{n}\right)=\left(\pi \cdot a_{1}, \ldots, \pi \cdot a_{n}\right)$. Note that $X^{\# 0}$ is 1 for any nominal set X. Henceforth we write $\boldsymbol{a}^{n}$, or simply $\boldsymbol{a}$ when $n$ is clear from the context, as a shorthand for a tuple $a_{1}, \ldots, a_{n}$ of distinct atoms, and further write $\left\{\boldsymbol{a}^{n}\right\}$ for the set $\left\{a_{1}, \ldots, a_{n}\right\}$. For pairs $\boldsymbol{a}, \boldsymbol{b} \in \mathbb{A}^{\# n}$ we define the multi-transposition ( $\left.\boldsymbol{a} \boldsymbol{b}\right)$ to be a fixed bijection on $\mathrm{A}$ such that $(\boldsymbol{a} \boldsymbol{b})\left(a_{i}\right)=b_{i}$ for all $1 \leq i \leq n$, and $(\boldsymbol{a} \boldsymbol{b})(c)=c$ for all $c \notin\{\boldsymbol{a}\} \cup\{\boldsymbol{b}\}$.

The separating tensor \# is closed and the associated internal-hom functor is denoted $[-,=]$. In particular, the internal homs $\left[\mathbb{A}^{\# n}, X\right]$, for $n \in \mathbb{N}$ and $X \in \mathbf{N o m}$, give rise to a notion of multi-atom abstraction. Indeed, the nominal set $\left[\mathbb{A}^{\# n}, X\right]$ has underlying set given by the quotient $\left|\mathbb{A}^{\# n} \times X\right| / \approx_{\alpha}$ under the $\alpha$-equivalence relation $\approx_{\alpha}$ defined as follows: $(\boldsymbol{a}, x) \approx_{\alpha}(\boldsymbol{b}, y)$ if and only if there exists a fresh $\boldsymbol{c} \in \mathbb{A}^{\# n}$ (i.e., a tuple $\boldsymbol{c} \in \mathbb{A}^{\# n}$ satisfying the condition $\boldsymbol{c} \# \boldsymbol{a}, x, \boldsymbol{b}, y)$ such that $(\boldsymbol{a} \boldsymbol{c}) \cdot x=(\boldsymbol{b} \boldsymbol{c}) \cdot y$. We write $\langle\boldsymbol{a}\rangle x$ for the equivalence class $[(\boldsymbol{a}, x)]_{\approx_{\alpha}}$. The nominal set $\left[\mathbb{A}^{\# n}, X\right]$ has action $\pi \cdot\langle\boldsymbol{a}\rangle x=\langle\pi \cdot \boldsymbol{a}\rangle \pi \cdot x$ Note that $\operatorname{supp}(\langle\boldsymbol{a}\rangle x)$ is $\operatorname{supp}(x) \backslash\{\boldsymbol{a}\}$.

5.2. Nominal Equational Systems. We specify a class of MESs on Nom, called Nominal Equational Systems (NESs).

Following (Clouston and Pitts, 2007) we define a nominal signature $\Sigma$ to be a family of nominal sets $\{\Sigma(n)\}_{n \in \mathbb{N}}$, each of which consists of the operators of arity $n$.

Example 5.1. The nominal signature $\Sigma_{\lambda}$ for the untyped $\lambda$-calculus is given by the nominal sets of operators

$$
\Sigma_{\lambda}(0)=\left\{\boldsymbol{V}_{a} \mid a \in \mathbb{A}\right\}, \quad \Sigma_{\lambda}(1)=\left\{\boldsymbol{L}_{a} \mid a \in \mathbb{A}\right\}, \quad \Sigma_{\lambda}(2)=\{\boldsymbol{A}\}
$$

with action

$$
\pi \cdot \boldsymbol{V}_{a}=\boldsymbol{V}_{\pi(a)}, \quad \pi \cdot \boldsymbol{L}_{a}=\boldsymbol{L}_{\pi(a)}, \quad \pi \cdot \boldsymbol{A}=\boldsymbol{A}
$$


To each nominal signature $\Sigma$, we associate the strong endofunctor $\left(F_{\Sigma}, \mathrm{st}^{\Sigma}\right)$ on $\underline{\text { Nom }}=$ (Nom, $1, \#,[-,=])$ as follows:

$$
\begin{array}{rcccc}
F_{\Sigma}(X)= & \coprod_{n \in \mathbb{N}} \Sigma(n) \times X^{n}, & & \\
\mathrm{st}_{X, Y}^{\Sigma}: & F_{\Sigma}(X) \# Y & \longrightarrow & F_{\Sigma}(X \# Y) \\
& & \left(\iota_{n}\left(\mathrm{o}, x_{1}, \ldots, x_{n}\right), y\right) & \longmapsto & \iota_{n}\left(\mathrm{o},\left(x_{1}, y\right), \ldots,\left(x_{n}, y\right)\right)
\end{array}
$$

for $X, Y \in \mathbf{N o m}$ and $n \in \mathbb{N}, \circ \in \Sigma(n), x_{1}, \ldots, x_{n} \in X, y \in Y$. Since Nom is cocomplete and the functor $F_{\Sigma}$ is $\omega$-cocontinuous, free $F_{\Sigma}$-algebras exist. The carrier of the free $F_{\Sigma}$-algebra $T_{\Sigma} X$ on $X$ has the following inductive syntactic description:

$$
\begin{aligned}
& t \in T_{\Sigma} X \quad::=x \quad(x \in X) \\
& \text { | o }\left(t_{1}, \ldots, t_{n}\right) \quad\left(o \in \Sigma(n), t_{1}, \ldots, t_{n} \in T_{\Sigma} X\right)
\end{aligned}
$$

with action given by $\pi \cdot x=\pi \cdot X x$ and $\pi \cdot \mathrm{o}\left(t_{1}, \ldots, t_{n}\right)=(\pi \cdot \mathrm{o})\left(\pi \cdot t_{1}, \ldots, \pi \cdot t_{n}\right)$. The associated term monad $\mathbf{T}_{\Sigma}=\left(T_{\Sigma}, \eta^{\Sigma}, \mu^{\Sigma}\right)$ is strong, with strength $\widehat{\text { st }}^{\Sigma}$ given as follows:

$$
\begin{array}{ccccc}
\widehat{\mathrm{st}}_{X, Y}^{\Sigma} & : & T_{\Sigma}(X) \# Y & \longrightarrow & T_{\Sigma}(X \# Y) \\
& : & (t, y) & \mapsto & t\{x \mapsto(x, y)\}_{x \in X}
\end{array}
$$

where $t\{x \mapsto(x, y)\}_{x \in X}$ denotes the term obtained by simultaneously substituting $(x, y)$ for each $x$ in the term $t$.

A NES is a MES $\left(\underline{\mathbf{N o m}}, \mathbf{T}_{\Sigma}, \mathcal{A}\right)$ for $\Sigma$ a nominal signature and $\mathcal{A}$ a set of equations with arities $\coprod_{i=1}^{\ell} \mathbb{A}^{\# n_{i}}$ for $\ell, n_{1}, \ldots, n_{\ell} \in \mathbb{N}$ and coarities $\mathbb{A}^{\# n}$ for $n \in \mathbb{N}$.

5.3. Nominal Equational Presentations. We introduce Nominal Equational Presentations (NEPs) as syntactic counterparts of NESs.

We define a variable context as a finite set of variables $|V|$ together with a function $V$ : $|V| \rightarrow \mathbb{N}$ assigning a valence to each variable. We write $x_{1}: n_{1}, \ldots, x_{\ell}: n_{\ell}$ for the variable context with variables $x_{1}, \ldots, x_{\ell}$ respectively of valence $n_{1}, \ldots, n_{\ell}$. Variable contexts are syntax for arities, and every such $V$ determines the arity

$$
\underline{V}=\coprod_{x \in|V|} \mathbb{A}^{\# V(x)}
$$

We write $x(\boldsymbol{a})$ for the element $\iota_{x}(\boldsymbol{a})$ of $\underline{V}$; when convenient, we further abbreviate $x()$ as $x$.

For $n \in \mathbb{N}$ and a variable context $V$, the following bijection

$$
\begin{aligned}
\operatorname{Nom} & \left(\mathbb{A}^{\# n}, T_{\Sigma} \underline{V}\right) \\
& \cong \operatorname{Nom}\left(1,\left[\mathbb{A}^{\# n}, T_{\Sigma} \underline{V}\right]\right) \\
& \cong\left\{\tau \in\left[\mathbb{A}^{\# n}, T_{\Sigma} \underline{V}\right] \mid \operatorname{supp}(\tau)=\emptyset\right\} \\
& =\left\{\langle\boldsymbol{a}\rangle t \in\left[\mathbb{A}^{\# n}, T_{\Sigma} \underline{V}\right] \mid \operatorname{supp}(t) \subseteq\{\boldsymbol{a}\}\right\}
\end{aligned}
$$

shows that a Kleisli map $\mathbb{A}^{\# n} \rightarrow T_{\Sigma} \underline{V}$ is determined by an $\alpha$-equivalence class $\langle\boldsymbol{a}\rangle t$ for $\boldsymbol{a} \in \mathbb{A}^{\# n}$ and $t \in\left(T_{\Sigma} \underline{V}\right)_{\boldsymbol{a}}=\left\{t \in T_{\Sigma} \underline{V} \mid \operatorname{supp}(t) \subseteq\{\boldsymbol{a}\}\right\}$. The set $\left(T_{\Sigma} \underline{V}\right)_{\boldsymbol{a}}$ has the following inductive syntactic description:

$$
\begin{array}{rll}
t \in\left(T_{\Sigma} \underline{V}\right)_{\boldsymbol{a}}::= & x(\boldsymbol{b}) & (x(\boldsymbol{b}) \in \underline{V} \text { such that }\{\boldsymbol{b}\} \subseteq\{\boldsymbol{a}\}) \\
& \mid \mathrm{o}\left(t_{1}, \ldots, t_{n}\right) & (\mathrm{o} \in \Sigma(n) \text { such that supp }(\mathrm{o}) \subseteq\{\boldsymbol{a}\}, \\
& & \text { and } \left.t_{1}, \ldots, t_{n} \in\left(T_{\Sigma} \underline{V}\right)_{\boldsymbol{a}}\right) .
\end{array}
$$


Directly motivated by this analysis, we define the notion of Nominal Equational Presentation (NEP) as follows. A nominal context $[\boldsymbol{a}] V$ consists of an atom context $\boldsymbol{a} \in \mathbb{A}^{\# n}$, for $n \in \mathbb{N}$, and a variable context $V$. A nominal term $t$, for a nominal signature $\Sigma$, in a nominal context $[\boldsymbol{a}] V$, denoted $[\boldsymbol{a}] V \vdash t$, is given by a term $t \in\left(T_{\Sigma} \underline{V}\right)_{\boldsymbol{a}}$; a nominal equation $[\boldsymbol{a}] V \vdash t \equiv t^{\prime}$ is given by a pair of nominal terms $t$ and $t^{\prime}$ in the same nominal context $[\boldsymbol{a}] V$. A NEP $\mathbb{T}=(\Sigma, E)$ consists of a nominal signature $\Sigma$ and a set of nominal equations $E$.

Example 5.2 (continued from Example 5.1, cf. (Gabbay and Mathijssen, 2007) and (Clouston and Pitts, 2007)). The NEP $\mathbb{T}_{\lambda}=\left(\Sigma_{\lambda}, E_{\lambda}\right)$ for $\alpha \beta \eta$-equivalence of untyped $\lambda$-terms has the following equations:

$$
\begin{array}{rrrl}
(\alpha) & {[a, b] x: 1} & \vdash & \boldsymbol{L}_{a} \cdot x(a) \equiv \boldsymbol{L}_{b} \cdot x(b) \\
\left(\beta_{\kappa}\right) & {[a] x: 0, y: 1} & \vdash & \boldsymbol{A}\left(\boldsymbol{L}_{a} \cdot x, y(a)\right) \equiv x \\
\left(\beta_{\boldsymbol{V}}\right) & {[a] x: 1} & \vdash & \boldsymbol{A}\left(\boldsymbol{L}_{a} \cdot \boldsymbol{V}_{a}, x(a)\right) \equiv x(a) \\
\left(\beta_{\boldsymbol{L}}\right) & {[a, b] x: 2, y: 1} & \vdash & \boldsymbol{A}\left(\boldsymbol{L}_{a} \cdot \boldsymbol{L}_{b} \cdot x(a, b), y(a)\right) \equiv \boldsymbol{L}_{b} \cdot \boldsymbol{A}\left(\boldsymbol{L}_{a} \cdot x(a, b), y(a)\right) \\
\left(\beta_{\boldsymbol{A}}\right) & {[a] x: 1, y: 1, z: 1} & & \boldsymbol{A}\left(\boldsymbol{L}_{a} \cdot \boldsymbol{A}(x(a), y(a)), z(a)\right) \\
& & & \equiv \boldsymbol{A}\left(\boldsymbol{A}\left(\boldsymbol{L}_{a} \cdot x(a), z(a)\right), \boldsymbol{A}\left(\boldsymbol{L}_{a} \cdot y(a), z(a)\right)\right) \\
\left(\beta_{\varepsilon}\right) & {[a, b] x: 1} & \vdash & \boldsymbol{A}\left(\boldsymbol{L}_{a} \cdot x(a), \boldsymbol{V}_{b}\right) \equiv x(b) \\
(\eta) & {[a] x: 0} & \vdash & \boldsymbol{L}_{a} \cdot \boldsymbol{A}\left(x, \boldsymbol{V}_{a}\right) \equiv x
\end{array}
$$

where we write $\boldsymbol{L}_{a} . t$ for $\boldsymbol{L}_{a}(t)$.

By construction, thus, NEPs represent NESs. Indeed, a NEP $\mathbb{T}=(\Sigma, E)$ determines the NES $\underline{\mathbb{T}}=\left(\underline{\mathbf{N o m}}, \mathbf{T}_{\Sigma}, \underline{E}\right)$ with the set of equations $\underline{E}$ given by

$$
\left\{\underline{[\boldsymbol{a}] V \vdash l} \equiv \underline{[\boldsymbol{a}] V \vdash r}: \mathbb{A}^{\# n} \rightarrow T_{\Sigma} \underline{V}\right\}([\boldsymbol{a}] V \vdash l \equiv r) \in E
$$

where $[\boldsymbol{a}] V \vdash t$ is the Kleisli map corresponding to $\langle\boldsymbol{a}\rangle t$ via the bijection (5.1).

5.4. Model theory. The model theory of a NEP $\mathbb{T}=(\Sigma, E)$ is derived from that of the NES $\mathbb{T}$. This we now spell out in elementary terms.

A $\mathbb{T}$-algebra is an $F_{\Sigma}$-algebra $\left(M, \mathrm{e}: F_{\Sigma} M \rightarrow M\right)$ such that for all nominal equations $([\boldsymbol{a}] V \vdash l \equiv r) \in E$,

$$
\llbracket[\boldsymbol{a}] V \vdash l \rrbracket_{(M, \mathrm{e})}=\llbracket[\boldsymbol{a}] V \vdash r \rrbracket_{(M, \mathrm{e})}: \llbracket[\boldsymbol{a}] V \rrbracket(M) \rightarrow M
$$

where

$$
\llbracket[\boldsymbol{a}] V \rrbracket(M)=\left(\prod_{x \in|V|}\left[\mathbb{A}^{\# V(x)}, M\right]\right) \# \mathbb{A}^{\# n}
$$

and where $\llbracket[\boldsymbol{a}] V \vdash t \rrbracket_{(M, \mathrm{e})}$ is inductively defined as follows:

- $\llbracket[\boldsymbol{a}] V \vdash x(\boldsymbol{b}) \rrbracket_{(M, \mathrm{e})}\left(\left\{\left\langle\boldsymbol{c}_{x}\right\rangle m_{x}\right\}_{x \in|V|}, \boldsymbol{d}\right)=\left(\boldsymbol{c}_{x} \boldsymbol{c}\right) \cdot m_{x}$ for $\boldsymbol{c}=(\boldsymbol{a} \boldsymbol{d}) \cdot \boldsymbol{b}$,

- $\llbracket[\boldsymbol{a}] V \vdash \mathrm{o}\left(t_{1}, \ldots, t_{k}\right) \rrbracket_{(M, \mathrm{e})}\left(\left\{\left\langle\boldsymbol{c}_{x}\right\rangle m_{x}\right\}_{x \in|V|}, \boldsymbol{d}\right)=\mathrm{e}_{k}\left(\mathrm{o}^{\prime}, t_{1}^{\prime}, \ldots, t_{k}^{\prime}\right)$ for $\mathrm{e}_{k}: \Sigma(k) \times M^{k} \rightarrow M$ the $k$-component of the structure map e, and

$$
\mathrm{o}^{\prime}=(\boldsymbol{a} \boldsymbol{d}) \cdot \mathrm{o}, \quad t_{i}^{\prime}=\llbracket[\boldsymbol{a}] V \vdash t_{i} \rrbracket_{(M, \mathrm{e})}\left(\left\{\left\langle\boldsymbol{c}_{x}\right\rangle m_{x}\right\}_{x \in|V|}, \boldsymbol{d}\right) .
$$

The category $\mathbb{T}$-Alg is the full subcategory of $F_{\Sigma}$-Alg consisting of $\mathbb{T}$-algebras. $\mathbb{T}$-Alg and $\underline{\mathbb{T}}$-Alg are isomorphic by construction. 


$$
\begin{aligned}
& \text { Eqvar } \frac{\left[\boldsymbol{a}^{n}\right] V \vdash_{E} t \equiv t^{\prime}}{\left[\boldsymbol{b}^{n}\right] V \vdash_{E}\left(\boldsymbol{a}^{n} \boldsymbol{b}^{n}\right) \cdot t \equiv\left(\boldsymbol{a}^{n} \boldsymbol{b}^{n}\right) \cdot t^{\prime}} \\
& \operatorname{Ref} \frac{[\boldsymbol{a}] V \vdash_{E} t}{[\boldsymbol{a}] V \vdash_{E} t \equiv t} \quad \operatorname{Sym} \frac{[\boldsymbol{a}] V \vdash_{E} t \equiv t^{\prime}}{[\boldsymbol{a}] V \vdash_{E} t^{\prime} \equiv t} \quad \operatorname{Trans} \frac{[\boldsymbol{a}] V \vdash_{E} t \equiv t^{\prime}[\boldsymbol{a}] V \vdash_{E} t^{\prime} \equiv t^{\prime \prime}}{[\boldsymbol{a}] V \vdash_{E} t \equiv t^{\prime \prime}} \\
& \text { Axiom } \frac{([\boldsymbol{a}] V \vdash l \equiv r) \in E}{[\boldsymbol{a}] V \vdash_{E} l \equiv r} \quad \operatorname{Elim} \frac{[\boldsymbol{a}, \boldsymbol{b}] V \vdash_{E} t \equiv t^{\prime}}{[\boldsymbol{a}] V \vdash_{E} t \equiv t^{\prime}}\left(\boldsymbol{b} \# \boldsymbol{a}, t, t^{\prime}\right) \\
& \text { Intro } \frac{[\boldsymbol{a}] V \vdash_{E} t \equiv t^{\prime}}{\left[\boldsymbol{a}, \boldsymbol{b}^{m}\right] V^{\langle m\rangle} \vdash_{E} t\left\{x\left(\boldsymbol{b}_{x}\right) \longmapsto x\left(\boldsymbol{b}_{x}, \boldsymbol{b}\right)\right\}_{x \in|V|} \equiv t^{\prime}\left\{x\left(\boldsymbol{b}_{x}\right) \longmapsto x\left(\boldsymbol{b}_{x}, \boldsymbol{b}\right)\right\}_{x \in|V|}} \\
& \text { with } \boldsymbol{b} \# \boldsymbol{a} \text { and } \forall_{x \in|V|} \boldsymbol{b} \# \boldsymbol{b}_{x} \\
& \text { where }\left|V^{\langle m\rangle}\right|=|V| \text { and } \forall_{x \in|V|} V^{\langle m\rangle}(x)=V(x)+m \\
& \text { Subst }_{\amalg} \frac{[\boldsymbol{a}] V \vdash_{E} t \equiv t^{\prime} \quad\left[\boldsymbol{b}_{x}{ }^{V(x)}\right] U \vdash_{E} s_{x} \equiv s_{x}^{\prime}(x \in|V|)}{[\boldsymbol{a}] U \vdash_{E} t\left\{x\left(\boldsymbol{b}_{x}\right) \longmapsto s_{x}\right\}_{x \in|V|} \equiv t^{\prime}\left\{x\left(\boldsymbol{b}_{x}\right) \longmapsto s_{x}^{\prime}\right\}_{x \in|V|}}
\end{aligned}
$$

Figure 1: Rules of SNEL.

Example 5.3 (continued from Example 5.2). A $\mathbb{T}_{\lambda}$-algebra has a carrier $M \in$ Nom with structure maps

$$
\llbracket \boldsymbol{V} \rrbracket: \mathbb{A} \rightarrow M, \quad \llbracket \boldsymbol{L} \rrbracket: \mathbb{A} \times M \rightarrow M, \quad \llbracket \boldsymbol{A} \rrbracket: M^{2} \rightarrow M
$$

satisfying the equations of the theory. For instance, according to the equation $(\alpha)$, we have that

$$
\llbracket \boldsymbol{L} \rrbracket(a,(c a) \cdot m)=\llbracket \boldsymbol{L} \rrbracket(b,(c b) \cdot m) \quad \text { for all }(\langle c\rangle m,(a, b)) \in[\mathbb{A}, M] \# \mathbb{A}^{\# 2}
$$

and, according to the equation $(\eta)$, we have that

$$
\llbracket \boldsymbol{L} \rrbracket(a, \llbracket \boldsymbol{A} \rrbracket(m, \llbracket \boldsymbol{V} \rrbracket(a)))=m \quad \text { for all }(m, a) \in M \# \mathbb{A} \text {. }
$$

5.5. Synthetic Nominal Equational Logic. For a NEP $\mathbb{T}=(\Sigma, E)$, we consider the EML associated to the NES $\mathbb{T}$ in syntactic form, and thereby synthesise a deductive system for deriving valid nominal equations in $\mathbb{T}$-algebras. The resulting Synthetic Nominal Equational Logic (SNEL) has the inference rules given in Figure 1. The substitution operation in the rules Intro and Subst $\amalg$ maps

$$
t \in T_{\Sigma} \underline{U}, \quad\left\{\left\langle\boldsymbol{c}_{x}\right\rangle s_{x} \in\left[\mathbb{A}^{\# U(x)}, T_{\Sigma} X\right]\right\}_{x \in|U|}
$$

to the nominal term

$$
t\left\{x\left(\boldsymbol{c}_{x}\right) \longmapsto s_{x}\right\}_{x \in|U|} \in T_{\Sigma} X
$$

defined by structural induction on $t$ as follows: 


$$
\begin{aligned}
x(\boldsymbol{a})\left\{x\left(\boldsymbol{c}_{x}\right) \longmapsto s_{x}\right\}_{x \in|U|} & =\left(\boldsymbol{c}_{x} \boldsymbol{a}\right) \cdot s_{x} \\
\mathrm{o}\left(t_{1}, \ldots, t_{k}\right)\left\{x\left(\boldsymbol{c}_{x}\right) \longmapsto s_{x}\right\}_{x \in|U|} & =\mathrm{o}\left(t_{1}\left\{x\left(\boldsymbol{c}_{x}\right) \longmapsto s_{x}\right\}_{x \in|U|}, \ldots, t_{k}\left\{x\left(\boldsymbol{c}_{x}\right) \longmapsto s_{x}\right\}_{x \in|U|}\right) .
\end{aligned}
$$

Remark 5.4. Note that under the rule Ref, the rules Intro and Subst $t_{\amalg}$ are inter-derivable with the rule

$$
\text { IntroSubst }_{\amalg} \frac{[\boldsymbol{a}] V \vdash_{E} t \equiv t^{\prime} \quad\left[\boldsymbol{b}_{x}^{V(x)}, \boldsymbol{b}\right] U \vdash_{E} s_{x} \equiv s_{x}^{\prime}(x \in|V|)}{[\boldsymbol{a}, \boldsymbol{b}] U \vdash_{E} t\left\{x\left(\boldsymbol{b}_{x}\right) \longmapsto s_{x}\right\}_{x \in|V|} \equiv t^{\prime}\left\{x\left(\boldsymbol{b}_{x}\right) \longmapsto s_{x}^{\prime}\right\}_{x \in|V|}}(\boldsymbol{b} \# \boldsymbol{a})
$$

Indeed, the above arises from the rule Intro for $\boldsymbol{b}$ on the judgement $[\boldsymbol{a}] V \vdash_{E} t \equiv t^{\prime}$ followed by the rule Subst ${ }_{\amalg}$ with respect to the family $\left[\boldsymbol{b}_{x} V(x), \boldsymbol{b}\right] U \vdash_{E} s_{x} \equiv s_{x}^{\prime}(x \in|V|)$; whilst, conversely, the rule Subst $\amalg$ is the special case of the rule IntroSubst $\amalg$ for $\boldsymbol{b}$ the empty tuple and the rule Intro arises by instantiating the rule IntroSubst $\amalg$ with the family $\left[\boldsymbol{b}_{x}{ }^{V(x)}, \boldsymbol{b}^{m}\right] V^{\langle m\rangle} \vdash_{E} x\left(\boldsymbol{b}_{x}, \boldsymbol{b}\right) \equiv x\left(\boldsymbol{b}_{x}, \boldsymbol{b}\right)(x \in|V|)$.

We also note that the rule Elim is in fact reversible, as the instantiation of the rule IntroSubst $_{\amalg}$ with the family $\left[\boldsymbol{b}_{x}{ }^{V(x)}, \boldsymbol{b}\right] V \vdash_{E} x\left(\boldsymbol{b}_{x}\right) \equiv x\left(\boldsymbol{b}_{x}\right)(x \in|V|)$ yields the derivability of the rule

$$
\operatorname{lnc} \frac{[\boldsymbol{a}] V \vdash_{E} t \equiv t^{\prime}}{[\boldsymbol{a}, \boldsymbol{b}] V \vdash_{E} t \equiv t^{\prime}}(\boldsymbol{b} \# \boldsymbol{a})
$$

Example 5.5 (continued from Example 5.3). We give a derivation of

$$
[a] x: 1, y: 0 \vdash \boldsymbol{A}\left(\boldsymbol{L}_{a} \cdot \boldsymbol{L}_{a} \cdot x(a), y\right) \equiv \boldsymbol{L}_{a} \cdot x(a)
$$

in the SNEL of $\mathbb{T}_{\lambda}$ :

$$
\begin{aligned}
& {[a, b] x: 1 \vdash \boldsymbol{L}_{a} \cdot x(a) \equiv \boldsymbol{L}_{b} \cdot x(b) \text { by Axiom }(\alpha)} \\
& \mathbf{A}: \frac{x \mapsto[c] x: 1, y: 0 \vdash x(c) \equiv x(c) \quad \text { by Ref }}{[a, b] x: 1, y: 0 \vdash \boldsymbol{L}_{a} \cdot x(a) \equiv \boldsymbol{L}_{b} \cdot x(b)} \text { by Subst } \amalg \\
& {[a, b] z: 2, w: 0 \vdash \boldsymbol{A}\left(\boldsymbol{L}_{a} \cdot z(a, b), w\right) \equiv \boldsymbol{A}\left(\boldsymbol{L}_{a} \cdot z(a, b), w\right) \text { by Ref }} \\
& z \mapsto[a, b] x: 1, y: 0 \vdash \boldsymbol{L}_{a} \cdot x(a) \equiv \boldsymbol{L}_{b} \cdot x(b) \quad \text { by } \mathbf{A} \\
& \mathbf{B}: \frac{w \longmapsto[] x: 1, y: 0 \vdash y \equiv y \quad \text { by Ref }}{[a, b] x: 1, y: 0 \vdash \boldsymbol{A}\left(\boldsymbol{L}_{a} . \boldsymbol{L}_{a} \cdot x(a), y\right) \equiv \boldsymbol{A}\left(\boldsymbol{L}_{a} \cdot \boldsymbol{L}_{b} \cdot x(b), y\right)} \text { by Subst }_{\amalg} \\
& \mathbf{C}: \frac{[a] x: 0, y: 1 \vdash \boldsymbol{A}\left(\boldsymbol{L}_{a} \cdot x, y(a)\right) \equiv x \quad \text { by Axiom }\left(\beta_{\kappa}\right)}{[a, b] x: 1, y: 2 \vdash \boldsymbol{A}\left(\boldsymbol{L}_{a} \cdot x(b), y(a, b)\right) \equiv x(b)} \text { by Intro } \\
& {[a, b] x: 1, y: 2 \vdash \boldsymbol{A}\left(\boldsymbol{L}_{a} \cdot x(b), y(a, b)\right) \equiv x(b) \text { by } \mathbf{C}} \\
& x \mapsto \quad[b] x: 1, y: 0 \vdash \boldsymbol{L}_{b} \cdot x(b) \equiv \boldsymbol{L}_{b} \cdot x(b) \quad \text { by Ref } \\
& \mathbf{D}: \frac{y \longmapsto[a, b] x: 1, y: 0 \vdash y \equiv y}{\text { by Ref }} \text { by Subst } \amalg \\
& \frac{[a, b] x: 1, y: 0 \vdash \boldsymbol{A}\left(\boldsymbol{L}_{a} \cdot \boldsymbol{L}_{a} \cdot x(a), y\right) \equiv \boldsymbol{L}_{a} \cdot x(a) \text { by Trans }(\operatorname{Trans}(\mathbf{B}, \mathbf{D}), \operatorname{Sym}(\mathbf{A}))}{[a] x: 1, y: 0 \vdash \boldsymbol{A}\left(\boldsymbol{L}_{a} \cdot \boldsymbol{L}_{a} \cdot x(a), y\right) \equiv \boldsymbol{L}_{a} \cdot x(a)} \text { by Elim }
\end{aligned}
$$


5.6. Soundness. By construction, if a nominal equation $[\boldsymbol{a}] V \vdash_{E} t \equiv t^{\prime}$ is derivable in SNEL, then the equation $\underline{E} \vdash \underline{[a}] V \vdash t \equiv \underline{a}] V \vdash t^{\prime}$ is derivable in EML. We explain why this is so for each rule.

- The SNEL rule Eqvar arises from the fact that

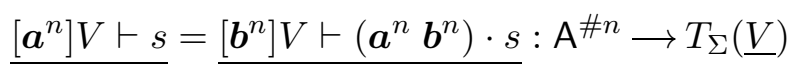

for all $\boldsymbol{b}^{n} \in \mathbb{A}^{\# n}$ and nominal terms $\left[\boldsymbol{a}^{n}\right] V \vdash s \underline{1}$

- The SNEL rules Ref, Sym, Trans, and Axiom directly mimic the corresponding EML rules.

- The SNEL rule Elim arises from the EML rule Local ${ }_{1}$ with respect to the epimorphic projection map $\mathbb{A}^{\#(n+m)} \longrightarrow \mathbb{A}^{\# n}$ sending $\left(\boldsymbol{a}^{n}, \boldsymbol{b}^{m}\right)$ to $\left(\boldsymbol{a}^{n}\right)$.

- The SNEL rule Intro arises from the EML rule Ext extended with the nominal set $\mathbb{A}^{\# m}$. Note that for $[\boldsymbol{a}] V \vdash s$, one has that $\left[\boldsymbol{a}^{n}, \boldsymbol{b}^{m}\right] V^{\langle m\rangle} \vdash s\left\{x\left(\boldsymbol{c}_{x}\right) \longmapsto x\left(\boldsymbol{c}_{x}, \boldsymbol{b}\right)\right\}_{x \in|V|}$ amounts to the composite

$$
\mathbb{A}^{\#(n+m)} \cong \mathbb{A}^{\# m} \# \mathbb{A}^{\# n} \stackrel{\left\langle\mathbb{A}^{\# m}\right\rangle \underline{[\boldsymbol{a}] V \vdash s}}{\longrightarrow} T_{\Sigma}\left(\mathbb{A}^{\# m} \# \underline{V}\right) \cong T_{\Sigma}\left(\coprod_{x \in|V|} \mathbb{A}^{\#(V(x)+m)}\right) .
$$

- The SNEL rule Subst $\amalg$ arises from the EML rule

$$
\text { Subst }_{\amalg} \frac{\underline{[\boldsymbol{a}] U \vdash t} \equiv \underline{[\boldsymbol{a}] U \vdash t^{\prime}} \quad \underline{\left[\boldsymbol{b}_{x}\right] V \vdash s_{x}} \equiv \underline{\left[\boldsymbol{b}_{x}\right] V \vdash s_{x}^{\prime}}(x \in|U|)}{(\underline{[\boldsymbol{a}] U \vdash t})\left\{\left[\underline{\left[\boldsymbol{b}_{x}\right] V \vdash s_{x}}\right]_{x \in|U|}\right\} \equiv\left(\underline{[\boldsymbol{a}] U \vdash t^{\prime}}\right)\left\{\left[\underline{\left[\boldsymbol{b}_{x}\right] V \vdash s_{x}^{\prime}}\right]_{x \in|U|}\right\}}
$$

noting that $\left.(\underline{[\boldsymbol{a}] U \vdash t})\left\{\underline{\left[\left[\boldsymbol{b}_{x}\right] V \vdash s_{x}\right.}\right]_{x \in|U|}\right\}=\underline{[\boldsymbol{a}] V \vdash t\left\{x\left(\boldsymbol{b}_{x}\right) \mapsto s_{x}\right\}_{x \in|U|}}$.

Thus, the soundness of SNEL follows from that of EML.

5.7. Completeness. We provide a sound and complete rewriting-style deduction system for NEPs, referred to as Synthetic Nominal Rewriting (SNR), and establish the completeness of SNEL.

For every NEP $\mathbb{T}$, the associated NES $\mathbb{T}$ is inductive. Indeed, using that in Nom finite limits commute with filtered colimits and equivariant functions are epimorphic iff their underlying function is surjective, one sees that the endofunctor $F_{\Sigma}$ associated to a nominal signature $\Sigma$ preserves colimits of $\omega$-chains and epimorphisms. Moreover, since for every $n \in \mathbb{N}$, the right adjoint $\left[\mathbb{A}^{\# n},-\right]$ is also a left adjoint, it follows that, for every variable context $V$, the nominal set $\underline{V}$ is compact and projective.

For a NEP $\mathbb{T}=(\Sigma, E)$, we consider the construction (3.3) for the associated NES $\mathbb{T}$. Since the forgetful functor $|-|:$ Nom $\rightarrow$ Set creates colimits, we have the following explicit description. For a nominal set $X$, the nominal set $\left(T_{\Sigma} X\right)_{1}$ has as underlying set the quotient $\left|T_{\Sigma} X\right| / \approx_{1}$ under the equivalence relation $\approx_{1}$ on $\left|T_{\Sigma} X\right|$ generated by the following rule:

$$
\frac{\left(\left[\boldsymbol{a}^{n}\right] V \vdash l \equiv r\right) \in E}{\left(\left(\boldsymbol{a}^{n} \boldsymbol{b}^{n}\right) \cdot l\right)\left\{x\left(\boldsymbol{c}_{x}\right) \longmapsto s_{x}\right\}_{x \in|V|} \approx_{1}\left(\left(\boldsymbol{a}^{n} \boldsymbol{b}^{n}\right) \cdot r\right)\left\{x\left(\boldsymbol{c}_{x}\right) \longmapsto s_{x}\right\}_{x \in|V|}}
$$

for $\boldsymbol{b} \in \mathbb{A}^{\# n}$ and $\left\langle\boldsymbol{c}_{x}\right\rangle s_{x} \in\left[\mathbb{A}^{\# V(x)}, T_{\Sigma} X\right]$ such that $\boldsymbol{b} \#\left\langle\boldsymbol{c}_{x}\right\rangle s_{x}$ for all $x \in|V|$. The action

\footnotetext{
${ }^{1}$ The omission of this rule in the SNEL presented in Fiore and Hur (2008) is an oversight.
} 


$$
\begin{gathered}
\operatorname{Ref} \frac{t \in\left|T_{\Sigma} X\right|}{t \approx_{E} t} \quad \operatorname{Sym} \frac{t \approx_{E} t^{\prime}}{t^{\prime} \approx_{E} t} \quad \operatorname{Trans} \frac{t \approx_{E} t^{\prime} t^{\prime} \approx_{E} t^{\prime \prime}}{t \approx_{E} t^{\prime \prime}} \\
\operatorname{Inst} \frac{\left(\left[\boldsymbol{a}^{n}\right] V \vdash l \equiv r\right) \in E}{\left(\left(\boldsymbol{a}^{n} \boldsymbol{b}^{n}\right) \cdot l\right)\left\{x\left(\boldsymbol{c}_{x}\right) \longmapsto s_{x}\right\}_{x \in|V|} \approx_{E}\left(\left(\boldsymbol{a}^{n} \boldsymbol{b}^{n}\right) \cdot r\right)\left\{x\left(\boldsymbol{c}_{x}\right) \longmapsto s_{x}\right\}_{x \in|V|}}
\end{gathered}
$$

with $\boldsymbol{b} \in \mathbb{A}^{\# n}$ and $\left\langle\boldsymbol{c}_{x}\right\rangle s_{x} \in\left[\mathbb{A}^{\# V(x)}, T_{\Sigma} X\right]$ such that $\boldsymbol{b} \#\left\langle\boldsymbol{c}_{x}\right\rangle s_{x}$ for all $x \in|V|$

$$
\text { Cong } \frac{s_{1} \approx_{E} s_{1}^{\prime}, \ldots, s_{k} \approx_{E} s_{k}^{\prime}}{\mathrm{o}\left(s_{1}, \ldots, s_{k}\right) \approx_{E} \mathrm{o}\left(s_{1}^{\prime}, \ldots, s_{k}^{\prime}\right)}(\mathrm{o} \in \Sigma(k))
$$

Figure 2: Rules for $\approx_{E}$.

of $\left(T_{\Sigma} X\right)_{1}$ is given by $\pi \cdot[t]_{\approx_{1}}=[\pi \cdot t]_{\approx_{1}}$. The equivariant function $q_{0}: T_{\Sigma} X \rightarrow\left(T_{\Sigma} X\right)_{1}$ maps a nominal term $t$ to its equivalence class $[t]_{\approx_{1}}$.

The nominal set $\left(T_{\Sigma} X\right)_{n}$, for $n \geq 2$, has as underlying set the quotient $\left|T_{\Sigma} X\right| / \approx_{n}$ under the equivalence relation $\approx_{n}$ on $\left|T_{\Sigma} X\right|$ generated by the following rules:

$$
\frac{s \approx_{n-1} s^{\prime}}{s \approx_{n} s^{\prime}} \quad \frac{s_{1} \approx_{n-1} s_{1}^{\prime}, \ldots, s_{k} \approx_{n-1} s_{k}^{\prime}}{\mathrm{o}\left(s_{1}, \ldots, s_{k}\right) \approx_{n} \mathrm{o}\left(s_{1}^{\prime}, \ldots, s_{k}^{\prime}\right)}(\mathrm{o} \in \Sigma(k))
$$

The action of $\left(T_{\Sigma} X\right)_{n}$ is given by $\pi \cdot[t]_{\approx_{n}}=[\pi \cdot t]_{\approx_{n}}$. The equivariant function $q_{n-1}$ : $\left(T_{\Sigma} X\right)_{n-1} \rightarrow\left(T_{\Sigma} X\right)_{n}$ maps $[t]_{\approx_{n-1}}$ to $[t]_{\approx_{n}}$.

The nominal set $T_{\mathbb{T}} X$, being the colimit of the chain $\left\{q_{n}:\left(T_{\Sigma} X\right)_{n} \rightarrow\left(T_{\Sigma} X\right)_{n+1}\right\}_{n \geq 0}$, is given by $\left|T_{\mathbb{T}} X\right|=\left|\bar{T}_{\Sigma} X\right| /{\approx_{E}}_{E}$ with action $\pi \cdot[t]_{\approx_{E}}=[\pi \cdot t]_{\approx_{E}}$, for $\approx_{E}$ the equivalence relation on $\left|T_{\Sigma} X\right|$ specified by the rules of Figure 2. The quotient map $\mathrm{q}_{X}^{\mathbb{T}}: T_{\Sigma} X \rightarrow T_{\mathbb{T}} X$ sends a nominal term $t$ to its equivalence class $[t]_{\approx_{E}}$.

The rules Inst and Cong of Figure 2 can be merged into a single one to yield a rewritingstyle deduction system. Indeed, by an induction on the depth of proof trees, one shows that the relation $\approx_{E}$ coincides with the equivalence relation $\approx_{E}^{\mathrm{R}} \subseteq\left(T_{\Sigma} X\right)^{2}$ generated by the rule

$$
\mathbf{C}\left[\left(\left(\boldsymbol{a}^{n} \boldsymbol{b}^{n}\right) \cdot l\right)\left\{x\left(\boldsymbol{c}_{x}\right) \longmapsto s_{x}\right\}_{x \in|V|}\right] \approx_{E}^{\mathrm{R}} \mathbf{C}\left[\left(\left(\boldsymbol{a}^{n} \boldsymbol{b}^{n}\right) \cdot r\right)\left\{x\left(\boldsymbol{c}_{x}\right) \longmapsto s_{x}\right\}_{x \in|V|}\right]
$$

for $\left(\left[\boldsymbol{a}^{n}\right] V \vdash l \equiv r\right) \in E$ and $\mathbf{C}[-]$ a context with one hole and possibly with elements from $X$, and for $\boldsymbol{b} \in \mathbb{A}^{\# n}$ and $\left\langle\boldsymbol{c}_{x}\right\rangle s_{x} \in\left[\mathbb{A}^{\# V(x)}, T_{\Sigma} X\right]$ such that $\boldsymbol{b}^{n} \#\left\langle\boldsymbol{c}_{x}\right\rangle s_{x}$ for all $x \in|V|$. Henceforth, the rewriting of nominal terms by the rule (5.2) is referred to as Synthetic Nominal Rewriting (SNR).

Example 5.6 ( $c f$. the derivation given in Example 5.5). We give a derivation of

$$
\boldsymbol{A}\left(\boldsymbol{L}_{a} . \boldsymbol{L}_{a} \cdot x(a), y\right) \approx^{\mathrm{R}} \boldsymbol{L}_{a} \cdot x(a) \text { in } T_{\Sigma_{\lambda}}(\underline{x: 1, y: 0)}
$$


in the SNR of $\mathbb{T}_{\lambda}$ :

$$
\begin{aligned}
& \boldsymbol{A}\left(\boldsymbol{L}_{a} . \boldsymbol{L}_{a} \cdot x(a), y\right) \\
& \approx^{\mathrm{R}} \quad \boldsymbol{L}_{b} \cdot x(b) \\
& \approx^{\mathrm{R}} \quad \boldsymbol{L}_{a} \cdot x(a) . \\
& \text { by }(\alpha): \approx^{\mathrm{R}} \boldsymbol{A ( \boldsymbol { L } _ { a } \cdot [ \quad \boldsymbol { L } _ { a } \cdot x ( a ) \{ x ( c ) \mapsto x ( c ) \} ] , y )} \\
& \boldsymbol{A}\left(\boldsymbol{L}_{a} \cdot\left[\quad \boldsymbol{L}_{b} \cdot x(b)\{x(c) \mapsto x(c)\}\right], y\right) \\
& \approx^{\mathrm{R}} \boldsymbol{A}\left(\boldsymbol{L}_{a} \cdot \boldsymbol{L}_{b} \cdot x(b), y\right) \\
& \text { by }\left(\beta_{\kappa}\right): \approx^{\mathrm{R}} \begin{aligned}
{\left[\boldsymbol{A}\left(\boldsymbol{L}_{a} \cdot x, y(a)\right)\{x\right.} & \left.\left.\mapsto \boldsymbol{L}_{b} \cdot x(b) ; y(a) \mapsto y\right\}\right] \\
{\left[x \left\{x \mapsto \boldsymbol{L}_{b} \cdot x(b) ; y(a)\right.\right.} & \mapsto y\}]
\end{aligned} \\
& \text { by }(\alpha): \approx^{\mathrm{R}} \begin{array}{c}
{\left[\boldsymbol{L}_{b} \cdot x(b)\{x(c) \mapsto x(c)\}\right]} \\
{\left[\boldsymbol{L}_{a} \cdot x(a)\{x(c) \mapsto x(c)\}\right]}
\end{array}
\end{aligned}
$$

The soundness and completeness of SNR is established by means of the internal completeness of the NES $\underline{\mathbb{T}}$ :

$$
\begin{aligned}
& {\left[\boldsymbol{a}^{n}\right] V \vdash s \equiv t \text { is satisfied by all } \mathbb{T} \text {-algebras }} \\
& \Longleftrightarrow \underline{\mathbb{T}} \text {-Alg } \models\left[\underline{\left[\boldsymbol{a}^{n}\right] V \vdash s} \equiv \underline{\left[\boldsymbol{a}^{n}\right] V \vdash t}: \mathbb{A}^{\# n} \rightarrow T_{\Sigma} \underline{V}\right. \\
& \Longleftrightarrow \mathrm{q}_{\underline{\underline{V}}}^{\mathbb{T}} \circ \underline{\left[\boldsymbol{a}^{n}\right] V \vdash s}=\mathrm{q}_{\underline{\underline{T}}}^{\underline{\mathbb{T}}} \underline{\left[\boldsymbol{a}^{n}\right] V \vdash t}: \mathbb{A}^{\# n} \rightarrow T_{\underline{\mathbb{T}}} \underline{V} \\
& \Longleftrightarrow \mathrm{q}_{\underline{\underline{V}}}^{\underline{\mathbb{T}}}\left(\underline{\left[\boldsymbol{a}^{n}\right] V \vdash s}\left(\boldsymbol{a}^{n}\right)\right)=\mathrm{q}_{\underline{\underline{T}}}^{\underline{\mathbb{T}}}\left(\underline{\left[\boldsymbol{a}^{n}\right] V \vdash t}\left(\boldsymbol{a}^{n}\right)\right) \text { in }\left|T_{\underline{\mathbb{T}}} \underline{V}\right| / \approx_{E} \\
& \Longleftrightarrow[s]_{\approx_{E}}=[t]_{\approx_{E}} \text { in }\left|T_{\underline{\mathbb{I}}} \underline{V}\right| / \approx_{E} \\
& \Longleftrightarrow s \approx_{E} t \quad \text { in }\left|T_{\underline{\mathbb{T}}} \underline{V}\right| \\
& \Longleftrightarrow s \approx_{E}^{\mathrm{R}} t \quad \text { in }\left|T_{\mathbb{\mathbb { T }}} \underline{V}\right| \text {. }
\end{aligned}
$$

The completeness of SNEL follows, as for all $t, t^{\prime} \in\left(T_{\Sigma} \underline{U}\right)_{d}$ every proof of $t \approx_{E} t^{\prime}$ can be turned into a proof of $[\boldsymbol{d}] U \vdash_{E} t \equiv t^{\prime}$ in SNEL. In particular, concerning the rule Inst, for every $\left(\left[\boldsymbol{a}^{n}\right] V \vdash l \equiv r\right) \in E, \boldsymbol{b} \in \mathbb{A}^{\# n}$, and $s_{x} \in\left(T_{\Sigma} \underline{U}\right)_{\left(\boldsymbol{c}_{\boldsymbol{x}} V(x), \boldsymbol{c}\right)}(x \in|V|)$ with $\boldsymbol{b} \# \boldsymbol{c}$, one deduces

$$
[\boldsymbol{b}, \boldsymbol{c}] U \vdash_{E} t_{l} \equiv t_{r} \quad, \text { for } t_{u}=((\boldsymbol{a} \boldsymbol{b}) \cdot u)\left\{x\left(\boldsymbol{c}_{x}\right) \mapsto s_{x}\right\}_{x \in|V|},
$$

by means of the rules Axiom, Eqvar, Ref, IntroSubst ${ }_{\amalg}$; and subsequently derives $[\boldsymbol{d}] U \vdash_{E} t_{l} \equiv t_{r}$ by means of the rules Elim and/or Inc for any $\boldsymbol{d}$ such that $t_{l}, t_{r} \in\left(T_{\Sigma} \underline{U}\right)_{\boldsymbol{d}}$.

5.8. Related work. Algebraic structure and rewriting in a nominal setting have already been considered in the literature. Gabbay and Mathijssen (2006, 2007) and Clouston and Pitts (2007) introduced an essentially equivalent notion of nominal algebra and provided sound and complete equational logics for them, whilst Fernández et al. (2004) introduced nominal rewriting.

Our SNEL and the Nominal Equational Logic (NEL) of Clouston and Pitts (2007) are equivalent. Indeed, see Hur (2010, Section 8.2.6) for a translation between the equality judgements of SNEL and NEL that respects the corresponding satisfaction relations. Thus, by virtue of the associated completeness theorems, SNEL and NEL establish the same theorems under different syntactic formalisms. 
The Nominal Rewriting (NR) of (Fernández and Gabbay, 2007) appears to be a termrewriting version of NEL. However, it has the shortcoming of not being complete for nominal equational reasoning (see (Hur, 2010, Section 8.2.7)).

Our approach allows us to also put the Equational Logic for Binding Terms (ELBT) of Hamana (2006) in the nominal context. Whereas SNEL arises from an EML on Nom, ELBT arises from a related EML on the super-category Set ${ }^{\mathbb{I}}$, for $\mathbb{I}$ the category of finite sets and injections. Crucially, however, the embedding Nom $\longrightarrow$ Set $^{\mathbb{I}}$ does not preserve the epimorphic projection maps $p_{n, m}: \mathbb{A}^{\#(n+m)} \rightarrow \mathbb{A}^{\# n}(n \geq 0, m>0)$. Thus, the only essential difference between SNEL and ELBT is that the latter lacks the rule Elim (which arises from the EML rule Local $_{1}$ with respect to $p_{n, m}$ ) as it is unsound.

\section{CONCLUSION}

We have introduced a categorical framework for the synthesis of equational logics. This comprises a general abstract notion of equational presentation together with an equational deduction system that is sound for a canonical model theory. In this context, we have also introduced a mathematical methodology for establishing completeness. This is based on an internal strong completeness result that typically leads, through an analysis of the construction of free algebras, to a characterisation of satisfiability via a rewriting-style deduction system embedded within the equational deduction system.

Two applications of our theory and methodology were presented. They respectively provide a rational reconstruction of Birkhoff's Equational Logic and a novel nominal logic for reasoning about algebraic structure with name-binding operators. A further major application was given in Fiore and Hur (2010) with the synthesis of Second-Order Equational Logic: a deductive system for equational reasoning about languages with variable binding and parameterised metavariables (see also Fiore and Mahmoud (2010)).

The extension of the theory of this paper from the mono-sorted to the multi-sorted setting requires a more involved categorical theory (see Fiore (2008); Fiore and Hur (2008); Hur (2010)). A yet more comprehensive extension for a theory of rewriting modulo equations has also been developed.

\section{ACKNOWLEDGEMENT}

We are most grateful to Pierre-Louis Curien for the invitation to contribute to this volume, for his feedback on the first draft of the paper that helped to improve the presentation, and for his patience in waiting for this revised version.

\section{REFERENCES}

G. Birkhoff. On the structure of abstract algebras. Mathematical Proceedings of the Cambridge Philosophical Society, 31(4):433-454, 1935.

A. Burroni. Algèbres graphiques (sur un concept de dimension dans les langages formels). Cahiers de topologie et géométrie différentielle, XXII(3):249-265, 1981.

R. Clouston and A. Pitts. Nominal equational logic. In L. Cardelli, M. Fiore, and G. Winskel, editors, Computation, Meaning and Logic: Articles dedicated to Gordon Plotkin, volume 172 of Electronic Notes in Theoretical Computer Science, pages 223-257. Elsevier, 2007. 
C. Ehresmann. Esquisses et types des structures algébriques. Bul. Inst. Polit. Iasi, XIV, 1968.

M. Fernández and M. J. Gabbay. Nominal rewriting. Information and Computation, 205 (6):917-965, 2007.

M. Fernández, M. J. Gabbay, and I. Mackie. Nominal rewriting systems. In Proceedings of the Sixth ACM-SIGPLAN International Conference on Principles and Practice of Declarative Programming (PPDP'04), pages 108-119. ACM, 2004.

M. Fiore. Second-order and dependently-sorted abstract syntax. In Proceedings of the Twenty-Third Annual Symposium on Logic in Computer Science (LICS'08), pages 5768. IEEE Computer Society, 2008.

M. Fiore and C.-K. Hur. Term equational systems and logics. In Proceedings of the Twenty-Fourth Conference on the Mathematical Foundations of Programming Semantics (MFPS'08), volume 218 of Electronic Notes in Theoretical Computer Science, pages 171-192. Elsevier, 2008.

M. Fiore and C.-K. Hur. Second-Order Equational Logic. In Proceedings of the 19th EACSL Annual Conference on Computer Science Logic (CSL 2010), volume 6247 of Lecture Notes in Computer Science, pages 320-335. Springer-Verlag, 2010.

M. Fiore and C.-K. Hur. On the construction of free algebras for equational systems. Theoretical Computer Science, 410(18):1704-1729, 2009.

M. Fiore and O. Mahmoud. Second-order algebraic theories. In Proceedings of the 35th International Symposium on Mathematical Foundations of Computer Science (MFCS 2010), volume 6281 of Lecture Notes in Computer Science, pages 368-380. Springer-Verlag, 2010.

M. J. Gabbay and A. Mathijssen. Nominal algebra. In Proceedings of the Eighteenth Nordic Workshop on Programming Theory (NWPT'06), 2006.

M. J. Gabbay and A. Mathijssen. A formal calculus for informal equality with binding. In Proceedings of the Fourteenth Workshop on Logic, Language, Information and Computation (WoLLIC'07), pages 162-176. Springer-Verlag, 2007.

M. J. Gabbay and A. Pitts. A new approach to abstract syntax with variable binding. Formal Aspects of Computing, 13:341-363, 2001.

M. Hamana. An initial algebra approach to term rewriting systems with variable binders. Higher-Order and Symbolic Computation, 19(2-3):231-262. Springer, 2006.

C.-K. Hur. Categorical Equational Systems: Algebraic Models and Equational Reasoning. PhD thesis, Computer Laboratory, University of Cambridge, 2010.

G. M. Kelly and A. J. Power. Adjunctions whose counits are coequalizers, and presentations of finitary enriched monads. Journal of Pure and Applied Algebra, 89:163-179, 1993.

F. W. Lawvere. Functorial Semantics of Algebraic Theories. PhD thesis, Columbia University, 1963.

S. Mac Lane and I. Moerdijk. Sheaves in Geometry and Logic. Springer-Verlag, 1992.

A. J. Power. Enriched Lawvere theories. Theory and Applications of Categories, 6:83-93, 1999.

E. Robinson. Variations on algebra: Monadicity and generalisations of equational theories. Formal Aspects of Computing, 13(3-5):308-326, 2002. 


\section{Appendix A. Proof of Theorem 2.2}

Notation. For $f: V \otimes A \longrightarrow B$, we write $\bar{f}: A \longrightarrow[V, B]$ for the transpose of $f$ with respect to the adjunction $V \otimes-\dashv[V,-]$ and $\widehat{f}: V \rightarrow[A, B]$ for the transpose of $f$ with respect to the adjuction $-\otimes A \dashv[A,-]$.

Proof of Theorem 2.2. We establish the soundness of each EML rule; i.e., that every $\mathbb{S}$-algebra satisfying the premises of a rule also satisfies the conclusion. The soundness of the rules Ref, Sym, Trans, and Axiom is trivial. To show the soundness of the rule Subst, one uses that, for all $u_{1}: C \longrightarrow T B$ and $u_{2}: B \longrightarrow T A$,

$$
\llbracket u_{1}\left\{u_{2}\right\} \rrbracket_{(X, s)}=\llbracket u_{1} \rrbracket_{(X, s)} \circ\left(\overline{\llbracket u_{2} \rrbracket_{(X, s)}} \otimes C\right):[A, X] \otimes C \rightarrow X .
$$

To show the soundness of the rule Ext, one uses that, for all $u: C \rightarrow T A$,

$$
\llbracket\langle V\rangle u \rrbracket_{(X, s)}=\llbracket u \rrbracket_{(X, s)} \circ(\widehat{\mathbf{p}} \otimes C) \circ \widetilde{\alpha}_{[V \otimes A, X], V, C}^{-1}:[V \otimes A, X] \otimes(V \otimes C) \rightarrow X,
$$

where

$$
\mathbf{p}=\left(([V \otimes A, X] \otimes V) \otimes A \stackrel{\widetilde{\alpha}_{[V \otimes A, X], V, A}}{\longrightarrow}[V \otimes A, X] \otimes(V \otimes A) \stackrel{\epsilon_{X}^{V \otimes A}}{\longrightarrow} X\right) .
$$

Finally, to show the soundness of the rule Local, one uses that, for all $u: C \rightarrow T A$ and $e: C^{\prime} \rightarrow C$,

$$
\llbracket u \circ e \rrbracket_{(X, s)}=\llbracket u \rrbracket_{(X, s)} \circ([A, X] \otimes e):[A, X] \otimes C^{\prime} \rightarrow X
$$

and that, for every jointly epimorphic family $\left\{e_{i}: C_{i} \rightarrow C\right\}_{i \in I}$, the family $\left\{[A, X] \otimes e_{i}\right.$ : $\left.[A, X] \otimes C_{i} \longrightarrow[A, X] \otimes C\right\}_{i \in I}$ is also jointly epimorphic.

\section{Appendix B. Proof of Theorem 3.1}

We introduce several lemmas before proceeding to prove the theorem.

Notation. For $f: V \otimes A \longrightarrow B$, we write $\bar{f}: A \longrightarrow[V, B]$ for the transpose of $f$ with respect to the adjunction $V \otimes-\dashv[V,-]$ and $\widehat{f}: V \rightarrow[A, B]$ for the transpose of $f$ with respect to the adjuction $-\otimes A \dashv[A,-]$.

Lemma B.1. Let $\mathbb{S}=(\underline{\mathscr{C}}, \mathbf{T}, \mathcal{A})$ be a MES. For every $\mathbb{S}$-algebra $(X, s: T X \rightarrow X)$, the $T$-algebra $\left([V, X], s^{V}: T[V, X] \longrightarrow[V, X]\right)$, where $s^{V}$ is the transpose of

$$
\underline{s^{V}}=\left(V \otimes T[V, X] \stackrel{\text { st }_{V,[V, X]}}{\longrightarrow} T(V \otimes[V, X]) \stackrel{T\left(\epsilon_{X}^{V}\right)}{\longrightarrow} T X \stackrel{s}{\longrightarrow} X\right),
$$

is an $\mathbb{S}$-algebra.

Proof. That $\left([V, X], s^{V}\right)$ is an Eilenberg-Moore algebra follows from transposing the following identities:

(1) $\underline{s^{V}} \circ\left(V \otimes \eta_{[V, X]}\right)=\epsilon_{X}^{V}: V \otimes[V, X] \rightarrow X$,

(2) $\underline{s^{V}} \circ\left(V \otimes \mu_{[V, X]}\right)=\underline{s^{V}} \circ\left(V \otimes T\left(\underline{s^{V}}\right)\right): V \otimes T T[V, X] \rightarrow X$. 
To show that every equation in $\mathcal{A}$ is satisfied in $\left([V, X], s^{V}\right)$, one uses that, for all $w: C \rightarrow T A$,

$$
\llbracket w \rrbracket_{\left([V, X], s^{V}\right)}=\overline{\llbracket w \rrbracket_{(X, s)} \circ(\widehat{\mathbf{p}} \otimes C) \circ \alpha_{V,[A,[V, X]], C}^{-1}}:[A,[V, X]] \otimes C \rightarrow[V, X]
$$

where $\mathbf{p}$ denotes the composite

$$
(V \otimes[A,[V, X]]) \otimes A \stackrel{\alpha_{V,[A,[V, X]], A}}{\longrightarrow} V \otimes([A,[V, X]] \otimes A) \stackrel{V \otimes \epsilon_{[V, X]}^{A}}{\longrightarrow} V \otimes[V, X] \stackrel{\epsilon_{X}^{V}}{\longrightarrow} X .
$$

Lemma B.2. For $\mathbb{S}=(\underline{\mathscr{C}}, \mathbf{T}, \mathcal{A})$ a MES admitting free algebras, the free $\mathbb{S}$-algebra monad $\mathbf{T}_{\mathbb{S}}$ on $\mathscr{C}$ is strong. The components of the strength $\mathrm{st}^{\mathbb{S}}$ are given by the unique maps such that the following diagram commutes:

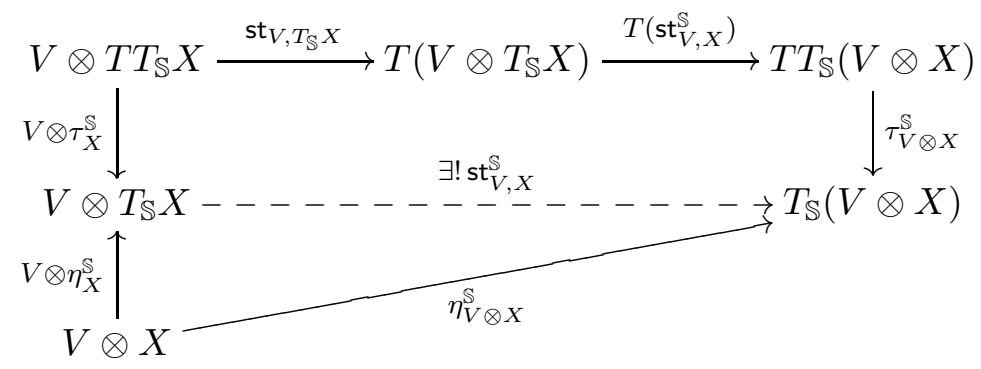

Proof. First note that $\overline{\mathrm{st}_{V, X}^{\mathbb{S}}}: T_{\mathbb{S}} X \rightarrow\left[V, T_{\mathbb{S}}(V \otimes X)\right]$ is the unique homomorphic extension of $\overline{\eta_{V \otimes X}^{\mathbb{S}}}: X \rightarrow\left[V, T_{\mathbb{S}}(V \otimes X)\right]$ with respect to the $\mathbb{S}$-algebra $\left(\left[V, T_{\mathbb{S}}(V \otimes X)\right],\left(\tau_{V \otimes X}^{\mathbb{S}}\right)^{V}\right)$.

The naturality of $\mathrm{st}^{\mathbb{S}}$ follows from the fact that, for all $f: V \rightarrow V^{\prime}$ and $g: C \longrightarrow C^{\prime}$, the maps

$$
\overline{T_{\mathbb{S}}(f \otimes g) \circ \mathrm{st}_{V, X}^{\mathbb{S}}}, \overline{\mathrm{st}_{V^{\prime}, X^{\prime}}^{\mathbb{S}} \circ\left(f \otimes T_{\mathbb{S}}(g)\right)}: T_{\mathbb{S}} X \rightarrow\left[V, T_{\mathbb{S}}\left(V^{\prime} \otimes X^{\prime}\right)\right]
$$

are both an homomorphic extension of $\overline{\eta_{V^{\prime} \otimes X^{\prime}}^{\mathbb{S}} \circ(f \otimes g)}: X \rightarrow\left[V, T_{\mathbb{S}}\left(V^{\prime} \otimes X^{\prime}\right)\right]$ with respect to the $\mathbb{S}$-algebra $\left(\left[V, T_{\mathbb{S}}\left(V^{\prime} \otimes X^{\prime}\right)\right],\left(\tau_{V^{\prime} \otimes X^{\prime}}^{\mathbb{S}}\right)^{V}\right)$.

Three of the four coherence conditions for strength follow from the fact that the maps

$$
\begin{aligned}
& \overline{T_{\mathbb{S}}\left(\lambda_{X}\right) \circ \mathrm{st}_{I, X}^{\mathbb{S}}}, \overline{\lambda_{T_{\mathbb{S}} X}}: T_{\mathbb{S}} X \rightarrow\left[I, T_{\mathbb{S}} X\right] \\
& \overline{T_{\mathbb{S}}\left(\alpha_{U, V, X}\right) \circ \mathrm{st}_{U \cdot V, X}^{\mathbb{S}}}, \overline{\mathrm{st}_{U, V \otimes X}^{\mathbb{S}} \circ\left(U \otimes \mathrm{st}_{V, X}^{\mathbb{S}}\right) \circ \alpha_{U, V, T_{\mathbb{S}} X}} \quad: \quad T_{\mathbb{S}} X \rightarrow\left[U \otimes V, T_{\mathbb{S}}(U \otimes(V \otimes X))\right] \\
& \overline{\mathrm{st}_{V, X}^{\mathbb{S}} \circ\left(V \otimes \mu_{X}^{\mathbb{S}}\right)}, \overline{\mu_{V \otimes X}^{\mathbb{S}} \circ T_{\mathbb{S}}\left(\mathrm{st}_{V, X}^{\mathbb{S}}\right) \circ \mathrm{st}_{V, T_{\mathbb{S}} X}^{\mathbb{S}}} \quad: \quad T_{\mathbb{S}} T_{\mathbb{S}} X \rightarrow\left[V, T_{\mathbb{S}}(V \otimes X)\right]
\end{aligned}
$$

are respectively homomorphic extensions of

$$
\begin{aligned}
\overline{\eta_{X}^{\mathbb{S}} \circ \lambda_{X}} & : X \rightarrow\left[I, T_{\mathbb{S}} X\right] \\
\overline{\eta_{U \otimes(V \otimes X)}^{\mathbb{S}}{ }^{\circ \alpha_{U, V, X}}} & : X \rightarrow\left[U \otimes V, T_{\mathbb{S}}(U \otimes(V \otimes X))\right] \\
\overline{\mathrm{st}_{X}^{\mathbb{S}}} & : \quad T_{\mathbb{S}} X \rightarrow\left[V, T_{\mathbb{S}}(V \otimes X)\right]
\end{aligned}
$$

with respect to the $\mathbb{S}$-algebras $\left(\left[I, T_{\mathbb{S}} X\right],\left(\tau_{X}^{\mathbb{S}}\right)^{I}\right),\left(\left[U \otimes V, T_{\mathbb{S}}(U \otimes(V \otimes X))\right],\left(\tau_{U \otimes(V \otimes X)}^{\mathbb{S}}\right)^{(U \otimes V)}\right)$, $\left(\left[V, T_{\mathbb{S}}(V \otimes X)\right],\left(\tau_{V \otimes X}^{\mathbb{S}}\right)^{V}\right)$. The remaining coherence condition is the triangle in the diagram above. 
Lemma B.3. Let $\mathbb{S}=(\underline{\mathscr{C}}, \mathbf{T}, \mathcal{A})$ be a MES admitting free algebras. Then, the quotient natural transformation $\mathrm{q}^{\mathbb{S}}: T \rightarrow T_{\mathbb{S}}$ is a strong functor morphism between the strong monads $\mathbf{T}$ and $\mathbf{T}_{\mathbb{S}}$. That is, the following diagram commutes:

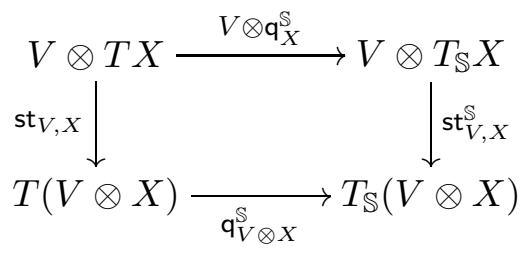

Proof. The commutativity of the diagram follows from the fact that both

$$
\overline{\mathrm{st}_{V, X}^{\mathbb{S}} \circ\left(V \otimes \mathrm{q}_{X}^{\mathbb{S}}\right)}, \overline{\mathrm{q}_{V \otimes X}^{\mathbb{S}} \circ \mathrm{st}_{V, X}}: T X \rightarrow\left[V, T_{\mathbb{S}}(V \otimes X)\right]
$$

are an homomorphic extension of $\overline{\eta_{V \otimes X}^{\mathbb{S}}}: X \rightarrow\left[V, T_{\mathbb{S}}(V \otimes X)\right]$ with respect to the EilenbergMoore algebra $\left(\left[V, T_{\mathbb{S}}(V \otimes X)\right],\left(\tau_{V \otimes X}^{\mathbb{S}}\right)^{V}\right)$ for the monad $\mathbf{T}$.

We are now ready to prove the internal completeness theorem.

Proof of Theorem 3.1. We show $1 \Rightarrow 2 \Rightarrow 3 \Rightarrow 1$.

$1 \Rightarrow$ 2. Holds vacuously.

2 2 3. Because, for all $w: C \rightarrow T A$, the $\operatorname{map} \mathrm{q}_{A}^{\mathbb{S}} \circ w: C \longrightarrow T_{\mathbb{S}} A$ factors as the composite

$$
\llbracket w \rrbracket_{\left(T_{\mathbb{S}} A, \tau_{A}^{\mathbb{S}}\right)} \circ(\widehat{\mathbf{p}} \otimes C) \circ \lambda_{C}^{-1}
$$

for $\mathbf{p}=\left(I \otimes A \stackrel{\lambda_{A}}{\longrightarrow} A \stackrel{\eta_{A}^{\mathbb{S}}}{\longrightarrow} T_{\mathbb{S}} A\right)$.

$3 \Rightarrow$ 1. Because for every $(X, s: T X \rightarrow X) \in \mathbb{S}$-Alg and $w: C \rightarrow T A$, the interpretation map $\llbracket w \rrbracket_{(X, s)}:[A, X] \otimes C \longrightarrow X$ factors as the composite

$$
s^{*} \circ T_{\mathbb{S}}\left(\epsilon_{X}^{A}\right) \circ \mathrm{st}_{[A, X], A}^{\mathbb{S}} \circ\left([A, X] \otimes\left(\mathbf{q}_{A}^{\mathbb{S}} \circ w\right)\right)
$$

where $s^{*}: T_{\mathbb{S}} X \rightarrow X$ is the unique homomorphic extension of the identity map on $X$ with respect to the $\mathbb{S}$-algebra $(X, s)$. 